\title{
ANALYSIS OF M-STATIONARY POINTS TO AN EPEC MODELING OLIGOPOLISTIC COMPETITION IN AN ELECTRICITY SPOT MARKET*
}

\author{
René Henrion ${ }^{1}$, Jiří Outrata ${ }^{2}$ And Thomas Surowiec ${ }^{3}$
}

\begin{abstract}
We consider an equilibrium problem with equilibrium constraints (EPEC) arising from the modeling of competition in an electricity spot market (under ISO regulation). For a characterization of equilibrium solutions, so-called M-stationarity conditions are derived. This first requires a structural analysis of the problem, e.g., verifying constraint qualifications. Second, the calmness property of a certain multifunction has to be verified in order to justify using M-stationarity conditions. Third, for stating the stationarity conditions, the coderivative of a normal cone mapping has to be calculated. Finally, the obtained necessary conditions are made fully explicit in terms of the problem data for one typical constellation. A simple two-settlement example serves as an illustration.
\end{abstract}

Mathematics Subject Classification. 90C30, 49J53.

Received September 6, 2009. Revised June 16, 2010 and November 4, 2010.

Published online January 19, 2011.

\section{INTRODUCTION}

In this article, we investigate a certain type of two-level game as it arises from a specific spot market model in power production. On the upper level of this game $n$ players wish to minimize their objectives $f_{i}$, by using a strategy $x_{i}$ from their respective set of admissible strategies $X_{i} \subseteq \mathbb{R}^{s}$. However, the value of $f_{i}$ is dependent also on the solution $z$ of a lower level optimization problem parameterized by the set of decisions of all (upperlevel) players. In this way, the players decisions are coupled implicitly. We consider a setting where the solution $z$ of the lower level optimization problem is characterized by a generalized equation

$$
0 \in F(x, z)+N_{C}(z)
$$

where $F \in \mathcal{C}^{1}\left(\mathbb{R}^{n s+t} ; \mathbb{R}^{t}\right)$ and $C \subseteq \mathbb{R}^{t}$ is a closed set. We will also make use of the common notation

$$
x_{-i}:=\left(x_{1}, \ldots, x_{i-1}, x_{i+1}, \ldots, x_{n}\right)
$$

\footnotetext{
Keywords and phrases. Equilibrium problems with equilibrium constraints, EPEC, M-stationary solutions, electricity spot market, calmness.

* This work was supported by the DFG Research Center MATHEON "Mathematics for key technologies" in Berlin and by the Grant Agency of the Czech Republic (Grant No. 201/09/1957).

${ }^{1}$ Weierstrass Institute for Applied Analysis and Stochastics, Mohrenstr. 39, 10117 Berlin, Germany. henrion@wias-berlin.de

${ }^{2}$ Institute of Information Theory and Automation, 18208 Praha 8, Czech Republic. outrata@utia.cas.cz

${ }^{3}$ Humboldt University Berlin, Unter den Linden 6, 10099 Berlin, Germany. surowiec@math.hu-berlin.de
} 
for the vector of decisions of player $i$ 's competitors. In order to simplify the notation, we adopt the convention $x=\left(x_{-i}, x_{i}\right)$ for all $i$.

Now, according to the previous remarks, each player $i$ solves an optimization problem of the type

$$
\min _{x_{i} \in X_{i}, z \in C}\left\{f_{i}\left(x_{i}, z\right) \mid 0 \in F\left(x_{-i}, x_{i}, z\right)+N_{C}(z)\right\} \quad(i=1, \ldots, n),
$$

where the competitors decisions $x_{-i}$ appear as exterior parameters. These optimization problems are not of standard type as they involve constraints which are solutions of another optimization problem themselves, i.e., a type equilibrium constraint. This in turn classifies the component problems as mathematical program with equilibrium constraints or an MPEC for short (see e.g., [15] or [22]). The collection (1.2) of all $n$ mutually coupled MPECs is called an equilibrium problem with equilibrium constraints or an EPEC for short. While the solution concept for an MPEC is obvious from the optimization context, a solution of the EPEC has to be interpreted in the sense of a Nash equilibrium. More precisely, we say that a vector $(\bar{x}, \bar{z})$ is a (local) solution to $(1.2)$, if for all $i=1, \ldots, n$, the pair $\left(\bar{x}_{i}, \bar{z}\right)$ belongs to the set of (local) solutions to the MPEC

$$
\min _{x_{i} \in X_{i}, z \in C}\left\{f_{i}\left(x_{i}, z\right) \mid 0 \in F\left(\bar{x}_{-i}, x_{i}, z\right)+N_{C}(z)\right\} .
$$

It should be mentioned here that EPECs may have a more general form than presented here. For instance, the objectives could also depend on the decisions of the competitors, as in a classical Nash-Cournot equilibrium, or (1.1) might represent some general equilibrium constraint, not just the solution set of an optimization problem. Our setting here is inspired by the application to the spot market model we have in mind.

The goal of this paper is to demonstrate how in a concrete application, one can use a certain type of stationarity conditions that EPEC solutions must necessarily satisfy. More precisely, we are dealing with socalled $M$-stationary points, which rely on first order necessary optimality conditions derived via the limiting normal cone as introduced by Mordukhovich (see [16] or [17] and the references therein). The usefulness of M-stationarity in the context of MPECs or EPECs has been widely demonstrated in the literature (e.g., $[4,17,20,21,28])$. We apply the methodology to an equilibrium problem in a so-called ISO regulated oligopolistic electricity market. Though the model is somewhat simplified in order to facilitate the analysis, we strive to keep the most meaningful facets intact. It is inspired by related work in $[6,11,12]$ and one that has recently been investigated in [7]. We also mention the interesting work in [26,27] devoted to EPECs/MPECs in market models though not related with M-stationarity as considered here.

Besides M- (or Mordukhovich-) stationarity, several other dual stationarity concepts are well established in the MPEC literature (e.g., C- (or Clarke-) stationarity and S- (or strong) stationarity). All these notions require the verification of certain constraint qualifications before they give rise to necessary optimality conditions. Although S-stationarity yields the strongest characterization among these, it suffers from the substantial drawback of relying on a restrictive constraint qualification which is hardly satisfied in our application. In contrast, in basically all situations of our application we are able to verify the constraint qualification needed for M-stationarity (calmness), which in turn yields a substantially stronger characterization of solutions than C-stationarity.

The paper is organized as follows: after an introducing some basic concepts needed in Section 2, we present the spot market model in Section 3 and analyze its basic structural properties in Section 4 . Section 5 then is devoted to M-stationarity conditions in general and to the verification of the needed constraint qualifications in the concrete setting of the spot market model. In Section 6, we show how the usage of a transformation formula for the Mordukhovich coderivative leads to the derivation of explicit M-stationarity conditions. Concrete formulae are provided in a special case and for a small example (two settlements) the issue of one producer becoming inactive in an equilibrium state is investigated. Finally, Section 7 presents a calmness result for a special non-polyhedral multifunction which is crucial for checking the constraint qualification mentioned above. As this result if of a general nature, it is left to the end of the text and can thus be considered as an independent contribution. 


\section{CONCEPTS AND TOOLS FROM VARIATIONAL ANALYSIS}

In this section, we provide some concepts and definitions facilitating our analysis. We begin with some stability concepts for multifunctions. Let $\Phi: \mathbb{R}^{s} \rightrightarrows \mathbb{R}^{t}$ be a multifunction, $(\bar{x}, \bar{z}) \in \operatorname{gph} \Phi$, where gph $\Phi:=$ $\{(x, z) \mid z \in \Phi(x)\}$. Then $\Phi$ is said to have the Aubin property around $(\bar{x}, \bar{z})$ provided there exists neighborhoods $\mathcal{U} \subseteq \mathbb{R}^{s}$ of $\bar{x}$ and $\mathcal{V} \subseteq \mathbb{R}^{t}$ of $\bar{z}$, and a constant $L>0$ such that

$$
d\left(z, \Phi\left(x^{\prime \prime}\right)\right) \leq L\left\|x^{\prime}-x^{\prime \prime}\right\| \quad \forall z \in \mathcal{V} \cap \Phi\left(x^{\prime}\right) \forall x^{\prime}, x^{\prime \prime} \in \mathcal{U}
$$

Here, $d(z, \Phi(\bar{x}))=\inf _{z^{\prime} \in \Phi(\bar{x})}\left\|z-z^{\prime}\right\|$. A weaker Lipschitz property is obtained when fixing one of the $x$-arguments as $\bar{x}$. More precisely we say that $\Phi$ is calm at $(\bar{x}, \bar{z})$ provided there exists neighborhoods $\mathcal{U} \subseteq \mathbb{R}^{s}$ of $\bar{x}$ and $\mathcal{V} \subseteq \mathbb{R}^{t}$ of $\bar{z}$, and a constant $L>0$ such that

$$
d(z, \Phi(\bar{x})) \leq L\left\|x^{\prime}-\bar{x}\right\|, \forall z \in \mathcal{V} \cap \Phi\left(x^{\prime}\right), \forall x^{\prime} \in \mathcal{U}
$$

Setting $\mathcal{V} \equiv \mathbb{R}^{t}$ in the previous definition corresponds to the so-called upper Lipschitzian property. Next, let $(\bar{x}, \bar{z})$ be a solution to the generalized equation (1.1) and define the multifunction $\Sigma: \mathbb{R}^{t} \rightrightarrows \mathbb{R}^{t}$ via a local partial linearization of (1.1)

$$
\Sigma(\xi):=\left\{z \in \mathbb{R}^{t} \mid \xi \in F(\bar{x}, \bar{z})+\nabla_{z} F(\bar{x}, \bar{z})(z-\bar{z})+N_{C}(z)\right\} .
$$

Suppose now that there exist neighborhoods $\mathcal{W}$ of $0 \in \mathbb{R}^{t}$ and $\mathcal{V}$ of $\bar{z}$ such that the map $\xi \mapsto \Sigma(\xi) \cap \mathcal{V}$ is single-valued and Lipschitz on $\mathcal{W}$ with modulus $\kappa$. Then (1.1) is called strongly regular at $(\bar{x}, \bar{z})$, with Lipschitz modulus $\kappa$. Given the solution mapping of (1.1)

$$
S(x):=\left\{z \in \mathbb{R}^{t} \mid 0 \in F(x, z)+N_{C}(z)\right\}
$$

we know from Robinson ([24], Thm. 2.1) that if (1.1) is strongly regular at $(\bar{x}, \bar{z})$, then for any $\varepsilon>0$ there exist neighborhoods $\mathcal{U}_{\varepsilon}$ of $\bar{x}$ and $\mathcal{V}_{\varepsilon}$ of $\bar{z}$ such that the mapping $x \mapsto S(x) \cap \mathcal{V}_{\varepsilon}$ is single-valued and Lipschitz on $\mathcal{U}_{\varepsilon}$ with Lipschitz modulus $(\kappa+\varepsilon) L$, where $L$ is the uniform Lipschitz modulus of $F(\cdot, z)$ on $\mathcal{U}_{\varepsilon}$ for all $z \in \mathcal{V}_{\varepsilon}$.

In the following, we introduce the Mordukhovich coderivative of a multifunction. Recall first that, for a closed set $C \subseteq \mathbb{R}^{t}$ and a point $\bar{z} \in C$, the contingent or Bouligand cone to $C$ at $\bar{z} \in C$ is defined as

$$
T_{C}(\bar{z}):=\operatorname{Limsup}_{\tau \searrow 0} \tau^{-1}(C-\bar{z})=\left\{d \in \mathbb{R}^{t} \mid \exists \tau_{k} \searrow 0, \exists d_{k} \rightarrow d: \forall k, \bar{z}+\tau_{k} d_{k} \in C\right\}
$$

Here, "Limsup" is to be understood as the upper limit of sets in the sense of Painlevé-Kuratowski (cf. [25]). Accordingly, the Fréchet normal cone to $C$ at $\bar{z} \in C$ comes as the dual to the contingent cone:

$$
\widehat{N}_{C}(\bar{z}):=\left[T_{C}(\bar{z})\right]^{\circ}
$$

Then the limiting or Mordukhovich normal cone to $C$ at $\bar{z} \in C$ is derived from the Fréchet normal cone in the following manner

$$
N_{C}(\bar{z}):=\operatorname{Limsup}_{\substack{z \rightarrow \bar{z} \\ z \in C}} \widehat{N}_{C}(z) .
$$

Using the Mordukhovich normal cone, we can lastly define the following coderivative for a set-valued function $\Phi$

$$
D^{*} \Phi(\bar{x}, \bar{z})\left(v^{*}\right)=\left\{x^{*} \in \mathbb{R}^{s} \mid\left(x^{*},-v^{*}\right) \in N_{\operatorname{gph} \Phi}(\bar{x}, \bar{z})\right\} \quad \forall v^{*} \in \mathbb{R}^{t}
$$

where $(\bar{x}, \bar{z}) \in \operatorname{gph} \Phi$. 


\section{AN EPEC MODELING COMPETITION IN AN ELECTRICITY SPOT MARKET}

We now present the spot market EPEC. Assume that the electricity network of interest is represented by a connected oriented graph with $m$ edges (transmission lines) and $N$ nodes. Throughout this paper, $B \in \mathbb{R}^{N \times m}$ is used to represent the incidence matrix of the electricity network, with entries

$$
b_{i j}=\left\{\begin{array}{cc}
1 & \text { if edge } j \text { enters node } i \\
-1 & \text { if edge } j \text { leaves node } i \\
0 & \text { otherwise. }
\end{array}\right.
$$

We assume that at each node, an electricity producer is located. In contrast, we allow some zero demands at some of the nodes under the (trivial) assumption that total network demand is strictly positive. The satisfaction of demand in each node of the network can be modeled as

$$
q+B y \geq d+L(y)
$$

Here, $y \in \mathbb{R}^{m}$ denotes the oriented flow vector of electricity along the edges of the graph and $d, q \in \mathbb{R}^{N}$ represent the vectors of demand and electricity generated at each node, respectively. In addition, the vector $L(y)$ collects the amount of electricity lost at the different nodes due to transmission for a given flow $y$. Thus, $q+B y-L(y)$ provides the vector of electricity available at the given nodes (= production + transmission - losses) explaining the relation (3.1). As noted in [6], it is reasonable to use the following explicit form for the loss function:

$$
L(y)=\left(\frac{1}{2} \sum_{j=1}^{m}\left|b_{1 j}\right| \rho_{j} y_{j}^{2}, \ldots, \frac{1}{2} \sum_{j=1}^{m}\left|b_{N j}\right| \rho_{j} y_{j}^{2}\right)^{T}
$$

Here, $\rho_{j} \geq 0$ is the loss coefficient of line $j$, for all $j=1, \ldots, m$. In this paper, we investigate both the case of positive losses, which is more realistic, but requires numerical procedures for the identification of EPEC solutions; and the loss-free case, which might be a good approximation only in networks with short transmission lines, yet it allows us to identify some explicit qualitative relations for EPEC solutions. Additionally, we impose the following bounds on production and flow

$$
0 \leq q_{i} \leq \hat{q}_{i} \quad(i=1, \ldots, N) \quad-\hat{y}_{j} \leq y_{j} \leq \hat{y}_{j} \quad(j=1, \ldots, m)
$$

Electricity spot market models are structured in such a way so that each of the competing generators bids a certain cost function to an independent system operator (ISO). In real spot markets, these cost functions are typically step functions which are difficult to cope with analytically. Therefore, following [11,12] (though the usage of quadratic bid functions goes back much further, see e.g., [3]), we assume cost functions are defined:

$$
c_{i}\left(\alpha_{i}, \beta_{i}, q_{i}\right)=\alpha_{i} q_{i}+\beta_{i} q_{i}^{2} \quad(i=1, \ldots, N) .
$$

Nevertheless, the linear and quadratic cost coefficients $\alpha_{i}$ and $\beta_{i}$ of the bidding functions may in reality differ from the true cost coefficients $\gamma_{i}$ and $\delta_{i}$, respectively, yet it is assumed that neither the ISO nor the other generators know the competitors' true cost coefficients. Hence, the ISO determines generation and flow such that demand is met in each node of the network and that the overall costs are minimized given the bid cost functions $c_{i}\left(\alpha_{i}, \beta_{i}, q_{i}\right)$

$$
\min _{q, y}\left\{\sum_{i=1}^{N} c_{i}\left(\alpha_{i}, \beta_{i}, q_{i}\right) \mid(q, y) \in G\right\}
$$

where

$$
G:=\left\{(q, y) \in \mathbb{R}^{N+m} \mid q+B y \geq d+L(y), 0 \leq q \leq \hat{q},-\hat{y} \leq y \leq \hat{y}\right\}
$$


The vector $(\alpha, \beta)$ appears only as a perturbation parameter in (3.3) and is therefore not considered a decision variable on this level. This parametric optimization problem is referred to as the ISO problem. For $\beta \in \mathbb{R}^{N}$ with $\beta_{i} \geq 0$ for $i=1, \ldots, N$, the objective function is convex. In such cases, we know that the corresponding optimal solutions $\left(q^{\prime}, y^{\prime}\right)$ are characterized as solutions of the following generalized equation arising from the KKT conditions of $(3.3)$

$$
0 \in\left(\begin{array}{c}
\alpha+2[\operatorname{diag} \beta] q \\
0
\end{array}\right)+N_{G}(q, y) .
$$

Here, we use $[\operatorname{diag} \beta]$ to denote the diagonal matrix with entries $\beta_{i}$ along the diagonal and $N_{G}$ to denote the normal cone in the sense of convex analysis.

In order to derive each generator's profit function, we need to know the clearing prices $\pi_{i}=\alpha_{i}+2 \beta_{i} q_{i}$ which are the derivatives of the corresponding cost function with respect to $q_{i}$. Now, the profit of generator $i$ is calculated to be the price times produced quantity of electricity minus production costs:

$$
f_{i}\left(\alpha_{i}, \beta_{i}, q, y\right)=\pi_{i} q_{i}-\gamma_{i} q_{i}-\delta_{i} q_{i}^{2}=\left(\alpha_{i}-\gamma_{i}\right) q_{i}+\left(2 \beta_{i}-\delta_{i}\right) q_{i}^{2} .
$$

In order to define our EPEC solution as a classical non-cooperative or Nash-Cournot equilibrium, we require that each generator $i$ solves the following mathematical program with equilibrium constraints (MPEC), which is formed from (3.4) by fixing the decisions of all other competitors

$$
\max _{\substack{\left(\alpha_{i}, \beta_{i}\right) \in \mathbb{R}^{2} \\
q, y}}\left\{f_{i}\left(\alpha_{i}, \beta_{i}, q, y\right) \mid 0 \in\left(\begin{array}{c}
\zeta\left(\alpha_{i}, \beta_{i}, q\right) \\
0
\end{array}\right)+N_{G}(q, y)\right\} \quad(i=1, \ldots, N),
$$

where $\zeta\left(\alpha_{i}, \beta_{i}, q\right):=\left(\bar{\alpha}_{-i}, \alpha_{i}\right)+2\left[\operatorname{diag}\left(\bar{\beta}_{-i}, \beta_{i}\right)\right] q$. Then the coupled system of MPECs sharing the same equilibrium constraint represents an equilibrium problem with equilibrium constraints (EPEC)

$$
\min _{\substack{\left(\alpha_{i}, \beta_{i}\right) \in \mathbb{R}^{2} \\
q, y}}\left\{-f_{i}\left(\alpha_{i}, \beta_{i}, q, y\right) \mid 0 \in\left(\begin{array}{c}
\alpha+2[\operatorname{diag} \beta] q \\
0
\end{array}\right)+N_{G}(q, y)\right\}(i=1, \ldots, N) .
$$

Defining

$$
F(\alpha, \beta, q, y):=\left(\begin{array}{c}
\alpha+2[\operatorname{diag} \beta] q \\
0
\end{array}\right),
$$

we can rewrite our spot market EPEC in the compact form

$$
\min _{\substack{\left(\alpha_{i}, \beta_{i}\right) \in \mathbb{R}^{2} \\ q, y}}\left\{-f_{i}\left(\alpha_{i}, \beta_{i}, q, y\right) \mid 0 \in F(\alpha, \beta, q, y)+N_{G}(q, y)\right\} \quad(i=1, \ldots, N) .
$$

In order to unburden our analysis from technical difficulties in very specific situations, we introduce a restricted class of solutions to (3.5). More precisely, we consider solutions $(\bar{\alpha}, \bar{\beta}, \bar{q}, \bar{y})$ such that

$$
\begin{array}{|cc|}
\hline \bar{\alpha}_{i}, \bar{\beta}_{i}>0 & i=1, \ldots, N \\
\bar{q}_{i}>0 & i=1, \ldots, l \\
\bar{q}_{i}=0 & i=l+1, \ldots, N \\
-\hat{y}_{j}<\bar{y}_{j}<\hat{y}_{j} & j=1, \ldots, k \\
\bar{y}_{j}=\hat{y}_{j} & j=k+1, \ldots, m \\
\hline
\end{array}
$$

As in [7], we only consider strictly positive bidding coefficients. Disregarding zero coefficients allows us to avoid economically nonsensical or pathological situations. In addition to the types of solutions analyzed in [7], we consider cases wherein certain transmission lines become congested, electricity is lost due to resistance, 
and some producers may not be participating at equilibrium. These considerations add a new level of difficulty to the analysis.

More precisely, we assume without loss of generality that only the first $l \geq 1$ generators are active. Note that $l=0$ is excluded since total demand is positive. Therefore, at least one producer must be active. The identification of situations where certain market participants are forced to become non-active seems to be of economic and at the same time of mathematical interest because it is here where the nonsmooth character of the problem emerges. It would only add to notational, not mathematical, difficulty to consider generation quantities reaching their respective upper bounds. This holds similarly for congestion. We therefore assume that the first $k$ transmission lines are uncongested, whereas transmission along the remaining ones reaches the upper capacity. Again, the consideration of lower capacities would only increase the notational complexity.

Finally, we mention that the focus of our presentation is not on a fully adequate description of the power flow model, but on the illustration of the M-stationarity concept for an EPEC carrying the main structural properties of an idealized ISO problem. Our model follows [6] and other references and ignores Kirchhoff's voltage law for simplifying the structural analysis. However, it does consider power loss along transmission lines. By adding Kirchhoff's voltage law (while ignoring power loss) one would arrive at the linearized DC power flow model (see, e.g., [10]).

\section{Structural PROperties of THE ISO PROBLEM}

In this section, we compile some structural properties of the ISO problem (3.3) required for the application of results on stationarity conditions appearing later in Section 5 .

For convenience, we partition $q=\left(q^{(1)}, q^{(2)}\right) \in \mathbb{R}^{l} \times \mathbb{R}^{N-l}$ and $y=\left(y^{(1)}, y^{(2)}\right) \in \mathbb{R}^{k} \times \mathbb{R}^{m-k}$. Given the feasible set $G$ from the ISO problem (3.3), it is easy to see that near solutions satisfying (3.6), $G$ can be described by

$$
G=\left\{(q, y) \in \mathbb{R}^{N+m} \mid H(q, y) \leq 0\right\} .
$$

Here, $H: \mathbb{R}^{N+m} \rightarrow \mathbb{R}^{2 N-l+m-k}$ is the twice continuously differentiable mapping defined by the inequalities that are active near solutions of the type given in (3.6)

$$
H(q, y):=\left(\begin{array}{c}
d+L(y)-q-B y \\
-q^{(2)} \\
y^{(2)}-\hat{y}^{(2)}
\end{array}\right) .
$$

In what follows, we will need some auxiliary statements, which easily follow from what is well known in the literature and which we compile into the next lemma.

Lemma 4.1. Let $B$ be any $(N, m)$-incidence matrix of some oriented connected graph. Then the following properties hold (with $\hat{y}$ referring to the vector of upper transmission bounds):

1. $\operatorname{ker} B^{T}=\mathbb{R}(1, \ldots, 1)^{T}$.

2. For any integer $p$ such that $1 \leq p \leq N$, each $(N-p, m)$-submatrix of $B$ has rank $N-p$.

3. $\forall \varepsilon>0, \exists \Delta^{\prime}>0, \forall \rho_{j} \in\left[0, \Delta^{\prime}\right), \forall y \in[-\hat{y}, \hat{y}]:\|\nabla L(y)\|<\varepsilon$.

4. $\exists \Delta^{\prime \prime}>0, \forall \rho_{j} \in\left[0, \Delta^{\prime \prime}\right), \forall y \in[-\hat{y}, \hat{y}]:$

$$
\text { if } \nabla^{T} L(y) z=B^{T} z \text { and } z_{i}=0 \text { for some } i \text { then } z=0 .
$$

Proof. For 1., see Biggs ([1], Prop. 4.3). In particular, rank $B=N-1$. For 2., assume that the rank of some $(N-p, m)$-submatrix of $B$ is smaller than $N-p$. Then, successively joining the $p$ left out rows to this submatrix and thus reconstructing $B$ can increase the rank at most by $p-1$, because the last row is already a linear combination of all the remaining $N-1$ rows (see 1.). Whence a contradiction with $\operatorname{rank} B=N-1$ (see 1 .). 3. is an immediate consequence of (3.2). Concerning 4., it follows from 3. that for small enough transmission losses, $\nabla L(y)$ can be considered arbitrarily small for all $y$ in the indicated compact range. Since rank $B=N-1$, 
one has $\operatorname{rank}(\nabla L(y)-B) \geq N-1$ for small losses. If this rank strictly increases, then the dimension of the corresponding kernel strictly decreases, hence $\operatorname{ker}\left(\nabla^{T} L(y)-B^{T}\right)=\{0\}$ by 1 . Otherwise, this rank remains $N-1$, hence the corresponding kernel stays one-dimensional. Now, by 1 . and a continuity argument there exists some $v \neq 0$, which can be chosen arbitrarily close to $(1, \ldots, 1)^{T}$ such that $\operatorname{ker}\left(\nabla^{T} L(y)-B^{T}\right)=\mathbb{R} v$. In either case, the asserted implication in 4 . follows.

The following lemma provides some initial properties of the constraint mapping $H$ defined in (4.2). In particular it clarifies under which conditions the inequality system (4.1) satisfies the Mangasarian-Fromovitz or Linear Independence Constraint Qualification (positive or complete linear independence of active gradients). Moreover, information pertaining to the Lagrange multipliers associated to (3.3) is derived. For clarity of presentation, we will need to split the matrix $\nabla L(y)-B$ into specific submatrices according to the parameters $l$ and $k$ of activity and congestion:

$$
\nabla L(y)-B=\left(\begin{array}{c}
\nabla L^{1}(y)-B^{1} \\
\nabla L^{2}(y)-B^{2}
\end{array}\right)=\left(\begin{array}{cc}
\nabla L^{11}(y)-B^{11} & \nabla L^{12}(y)-B^{12} \\
\nabla L^{21}(y)-B^{21} & \nabla L^{22}(y)-B^{22}
\end{array}\right)
$$

Lemma 4.2. Let $(\bar{\alpha}, \bar{\beta}, \bar{q}, \bar{y})$ be a solution to (3.5) satisfying (3.6). Then, there exists some $\Delta>0$ such that under the condition $\rho_{j} \in[0, \Delta)(j=1, \ldots, m)$ the following properties hold:

1. (a) The rows of $\nabla H(\bar{q}, \bar{y})$ are positively linearly independent.

(b) If $l=N$ (all generators active) or $k=m$ (no congestion) then $\nabla H(\bar{q}, \bar{y})$ is surjective.

(c) If $1 \leq l<N, 0 \leq k<m$, and $B^{21}$ is surjective, then $\nabla H(\bar{q}, \bar{y})$ is surjective.

2. $H(\bar{q}, \bar{y})=0$.

3. (a) Concerning the ISO problem (3.3) (with fixed parameters $(\bar{\alpha}, \bar{\beta})$ ), strict complementarity holds for the first $l$ demand-satisfaction constraints (3.1) (corresponding to active producers).

(b) If $k=m$ (no congestion) or all multipliers associated with the flow constraints $y^{(2)} \leq \hat{y}^{(2)}$ vanish, then strict complementarity holds for all demand-satisfaction constraints (3.1).

Proof. The Jacobian of the mapping $H(q, y)$ at $(\bar{q}, \bar{y})$ becomes

$$
\nabla H(\bar{q}, \bar{y})=\left(\begin{array}{cccc}
-I_{1} & 0 & \nabla L^{11}(\bar{y})-B^{11} & \nabla L^{12}(\bar{y})-B^{12} \\
0 & -I_{2} & \nabla L^{21}(\bar{y})-B^{21} & \nabla L^{22}(\bar{y})-B^{22} \\
0 & -I_{2} & 0 & 0 \\
0 & 0 & 0 & I_{3}
\end{array}\right) .
$$

Assume a relation $\nabla^{T} H(\bar{q}, \bar{y}) c=0$ for some $c \geq 0$

$$
\left(\begin{array}{cccc}
-I_{1} & 0 & 0 & 0 \\
0 & -I_{2} & -I_{2} & 0 \\
\left(\nabla L^{11}(\bar{y})-B^{11}\right)^{T} & \left(\nabla L^{21}(\bar{y})-B^{21}\right)^{T} & 0 & 0 \\
\left(\nabla L^{12}(\bar{y})-B^{12}\right)^{T} & \left(\nabla L^{22}(\bar{y})-B^{22}\right)^{T} & 0 & I_{3}
\end{array}\right)\left(\begin{array}{l}
c_{1} \\
c_{2} \\
c_{3} \\
c_{4}
\end{array}\right)=0 .
$$

Then

$$
c_{1}=0, c_{2}=-c_{3},\left(\nabla L^{21}(\bar{y})-B^{21}\right)^{T} c_{2}=0,\left(\nabla L^{22}(\bar{y})-B^{22}\right)^{T} c_{2}=-c_{4} .
$$

Given that $c_{2}, c_{3} \geq 0$, it follows that $c_{2}=c_{3}=0$, and so $c_{4}=0$ as well. Therefore, $c=0$, proving the positive linear independence of the rows of $\nabla H(\bar{q}, \bar{y})$ stated in 1.(a). Inspecting again (4.4), we observe, that the conclusion $c=0$ could equally well be drawn from the relation $\nabla^{T} H(\bar{q}, \bar{y}) c=0$ upon replacing the original assumption $c \geq 0$ by the injectivity of $\left(\nabla L^{21}(\bar{y})-B^{21}\right)^{T}$. This, however, follows from the assumed surjectivity of $B^{21}$ if $\Delta>0$ in the statement of our lemma is chosen small enough as to maintain surjectivity of $\nabla L^{21}(\bar{y})-B^{21}$ via statement 3. of Lemma 4.1. This shows 1.(c). Concerning 1.(b), consider the case $l=N$ first, which implies that the third row block of $\nabla H(\bar{q}, \bar{y})$ in $(4.3)$ is missing. Surjectivity of $\nabla H(\bar{q}, \bar{y})$ is obvious then. If in contrast $k=m$, then the fourth row block of $\nabla H(\bar{q}, \bar{y})$ in (4.3) is missing. Surjectivity of $\nabla H(\bar{q}, \bar{y})$ would follow now from 
the surjectivity of $\left(\nabla L^{2}(\bar{y})-B^{2}\right)$. As per Lemma 4.1 (statement 2.), $B^{2}$ has rank $N-l$. Then, as a consequence of Lemma 4.1 (statement 3.), there exists some $\Delta>0$ such that $\left(\nabla L^{2}(\bar{y})-B^{2}\right)$ has rank $N-l$ too whenever $\rho_{j} \in[0, \Delta)$, for all $j=1, \ldots, m$ and $\Delta>0$. In other words, $\left(\nabla L^{2}(\bar{y})-B^{2}\right)$ is surjective and 1.(b) is proven.

Statement 1.(a) guarantees the existence of Lagrange multipliers such that the first-order optimality conditions of (3.3) hold for a solution $(\bar{\alpha}, \bar{\beta}, \bar{q}, \bar{y})$. Accordingly, there exist $\bar{\lambda}^{1}, \bar{\lambda}^{2}, \bar{\mu}, \bar{\eta} \geq 0$ such that

$$
\begin{aligned}
\left(\begin{array}{c}
\bar{\alpha}+2[\operatorname{diag} \bar{\beta}] \bar{q} \\
0
\end{array}\right)+\nabla^{T} H(\bar{q}, \bar{y})\left(\begin{array}{c}
\bar{\lambda}^{(1)} \\
\bar{\lambda}^{(2)} \\
\bar{\mu} \\
\bar{\eta}
\end{array}\right) & =0 \\
H(\bar{q}, \bar{y}) \bullet\left(\bar{\lambda}^{1}, \bar{\lambda}^{2}, \bar{\mu}, \bar{\eta}\right) & =0 .
\end{aligned}
$$

Here, we let "•" denote the Hadamard, or component-wise, product. Then by taking into account (3.6) we obtain the following set of relations

$$
\begin{aligned}
0 & <\bar{\alpha}_{i}+2 \bar{\beta}_{i} \bar{q}_{i}=\bar{\lambda}_{i}^{(1)}, \quad i=1, \ldots, l \\
0 & <\bar{\alpha}_{i}=\bar{\lambda}_{i-l}^{(2)}+\bar{\mu}_{i-l}, \quad i=l+1, \ldots, N \\
0 & =\left(\nabla L^{11}(\bar{y})-B^{11}\right)^{T} \bar{\lambda}^{(1)}+\left(\nabla L^{21}(\bar{y})-B^{21}\right)^{T} \bar{\lambda}^{(2)} \\
-\eta & =\left(\nabla L^{12}(y)-B^{12}\right)^{T} \bar{\lambda}^{(1)}+\left(\nabla L^{22}(y)-B^{22}\right)^{T} \bar{\lambda}^{(2)} .
\end{aligned}
$$

From (4.7) we derive statement 3.(a). Under any of the assumptions of statement 3.(b), (4.9) and (4.10) combine to

$$
(\nabla L(\bar{y})-B)^{T}\left(\begin{array}{c}
\bar{\lambda}^{(1)} \\
\bar{\lambda}^{(2)}
\end{array}\right)=0
$$

Choosing $\Delta$ in the assertion of this lemma equal to $\Delta^{\prime \prime}$ in statement 4 . of Lemma 4.1, we may draw the following conclusion: if there existed $i \in\{l+1, \ldots, N\}$ such that $\bar{\lambda}_{i-l}^{(2)}=0$ then $\bar{\lambda}^{(1)}=\bar{\lambda}^{(2)}=0$, a contradiction with (4.7). Therefore, $\bar{\lambda}_{i-l}^{(2)}>0$ and statement 3.(b) follows.

In order to prove statement 2 , we first observe that $H_{i}(\bar{q}, \bar{y})=0$ for $i>N$ because by definition of $q^{(2)}$ and $y^{(2)}$ in (4.2) via (3.6), one has that $\bar{q}^{(2)}=0$ and $\bar{y}^{(2)}=\hat{y}^{(2)}$. Moreover, (4.7) along with (4.6) yields that $H_{i}(\bar{q}, \bar{y})=0$ for $i \leq l$. It remains to prove that $H_{i}(\bar{q}, \bar{y})=0$ for $i \in\{l+1, \ldots, N\}$. This would follow easily from (4.6) and the just proven statement 3.(b) of the lemma in the case of $k=m$ (no congestion). The case of congestion requires a more subtle reasoning, however. Therefore, assume there exists some $i^{*} \in\{l+1, \ldots, N\}$ such that $H_{i^{*}}(\bar{q}, \bar{y})<0$. Given the structure of $H$ and noting that the loss function $L$ has nonnegative components, this amounts to saying that

$$
0 \leq d_{i^{*}}+L_{i^{*}}(\bar{y})<\bar{q}_{i^{*}}+\sum_{j=1}^{m} b_{i^{*} j} \bar{y}_{j} .
$$

Recalling that $i^{*}$ is chosen among the set of inactive producers, we derive that

$$
\sum_{j=1}^{m} b_{i^{*} j} \bar{y}_{j}>0
$$

Now, for any $i^{1} \in\{1, \ldots, N\}$, we define $i^{2} \in\{1, \ldots, N\}$ to be a critical neighbor of $i^{1}$ if there exists some $j \in\{1, \ldots, m\}$ such that

$$
\left|b_{i^{1} j}\right|=\left|b_{i^{2} j}\right|=1, b_{i^{1} j} b_{i^{2} j}=-1, b_{i^{1} j} \bar{y}_{j}>0 .
$$

We define the set $\Pi \subseteq\{1, \ldots, N\}$ to consist of our fixed node $i^{*}$, all its critical neighbors, all the critical neighbors of these critical neighbors etc. Define $\Lambda \subseteq\{1, \ldots, m\}$ to consist of those transmission lines connecting nodes 
inside $\Pi$ only. Then, $\Pi \times \Lambda$ constitutes a subgraph of the original one which is connected again by construction (there exists a path from any node in $\Pi$ to $i^{*}$ ). Consequently, the associated submatrix of $B$ is again the incidence matrix of a connected oriented graph. Therefore, we can invoke statement 1. of Lemma 4.1 to conclude that $\sum_{i \in \Pi} b_{i j}=0$ for all $j \in \Lambda$. This in turn implies that

$$
\sum_{i \in \Pi} \sum_{j \in \Lambda} b_{i j} \bar{y}_{j}=0
$$

Next we observe that

$$
\sum_{j \in \Lambda^{c}} b_{i j} \bar{y}_{j} \leq 0 \quad \forall i \in \Pi .
$$

Indeed, otherwise there exists some $i \in \Pi$ and $j \in \Lambda^{c}$ with $b_{i j} \bar{y}_{j}>0$. In particular, $b_{i j} \neq 0$, whence $\left|b_{i j}\right|=1$. Moreover, let $i^{a} \in\{1, \ldots, N\}$ be the uniquely defined node such that $b_{i j} b_{i^{a} j}=-1$ (i.e., $i^{a}$ is the node joined with $i$ via edge $j$ ). Then, by definition, $i^{a}$ is a critical neighbor of $i$, whence $i^{a} \in \Pi$. Therefore, the edge $j$ joining $i$ and $i^{a}$ belongs to $\Lambda$ which contradicts $j \in \Lambda^{c}$. Now, combining (4.12) with (4.15) yields $\sum_{j \in \Lambda} b_{i^{*} j} \bar{y}_{j}>0$ which along with $i^{*} \in \Pi$ and (4.14) allows to infer the existence of some $i^{* *} \in \Pi$ such that $\sum_{j \in \Lambda} b_{i^{* *} j} \bar{y}_{j}<0$. Then, the demand satisfaction at $i^{* *}$ provides that (taking into account (4.15) for $i^{* *}$ )

$$
0 \leq d_{i^{* *}}+L_{i^{* *}}(\bar{y}) \leq \bar{q}_{i^{* *}}+\sum_{j=1}^{m} b_{i^{* * j}} \bar{y}_{j}<\bar{q}_{i^{* *}} .
$$

In other words, $i^{* *}$ is an active producer, hence $i^{* *} \leq l$. Finally, we observe, that for each critical neighbor $i^{\prime}$ of $i^{*}$ we may modify the flow vector $\bar{y}$ to some $\tilde{y}$ such that $(\bar{q}, \tilde{y})$ remains feasible for the ISO problem (3.3) (i.e., $H(\bar{q}, \tilde{y}) \leq 0)$ and that $H_{i^{\prime}}(\bar{q}, \tilde{y})<0$. Indeed, assuming that $i^{\prime}$ and $i^{*}$ are joined by some edge $j^{\prime}$, we may define $\tilde{y}$ as

$$
\tilde{y}_{k}:=\left\{\begin{array}{cc}
\bar{y}_{k} & k \neq j^{\prime} \\
\bar{y}_{j^{\prime}}-\varepsilon & k=j^{\prime}, b_{i^{*} j^{\prime}}=1 \\
\bar{y}_{j^{\prime}}+\varepsilon & k=j^{\prime}, b_{i^{*} j^{\prime}}=-1,
\end{array}\right.
$$

where $\varepsilon>0$ is chosen small enough to guarantee that the demand at node $i^{*}$ remains satisfied after the modification (which is possible by (4.11)):

$$
\bar{q}_{i^{*}}+\sum_{j=1}^{m} b_{i^{*} j} \tilde{y}_{j} \geq d_{i^{*}}+L_{i^{*}}(\bar{y}) .
$$

Moreover, let $\varepsilon>0$ be small enough such that $\tilde{y}_{j} \leq \hat{y}_{j}$. This is possible due to $\bar{y}_{j} \leq \hat{y}_{j}$ (by feasibility of $\bar{y}$ ) and upon observing that $\bar{y}_{j}>0$ in case of $b_{i^{*} j}=1$ and $\bar{y}_{j}<0$ in case of $b_{i^{*} j}=-1$ (see (4.13)). Thus, $\tilde{y}$ is feasible for the constraint $y \leq \hat{y}$. In addition, we have that $b_{i^{\prime} j^{\prime}} \tilde{y}_{j^{\prime}}=b_{i^{\prime} j^{\prime}} \bar{y}_{j^{\prime}}+\varepsilon$ by construction of $\tilde{y}_{j^{\prime}}$ and by $b_{i^{*} j^{\prime}}=-b_{i^{\prime} j^{\prime}}$. Then, the demand satisfaction at node $i^{\prime}$ reads as

$$
d_{i^{\prime}}+L_{i^{\prime}}(\bar{y}) \leq \bar{q}_{i^{\prime}}+\sum_{j=1}^{m} b_{i^{\prime} j} \bar{y}_{j}=\bar{q}_{i^{\prime}}+\sum_{j=1}^{m} b_{i^{\prime} j} \tilde{y}_{j}-\varepsilon .
$$

Since the demand satisfaction relations at nodes $i^{\prime}$ and $i^{*}$ are the only ones affected by the transition from $\bar{y}$ to $\tilde{y}$, it follows that $(\bar{q}, \tilde{y})$ remains feasible for $(3.3)$ (i.e., $H(\bar{q}, \tilde{y}) \leq 0)$ and $H_{i^{\prime}}(\bar{q}, \tilde{y})<0$ (demand is strictly exceeded by offer). In this way, we have shifted the strict inequality $H_{i^{*}}(\bar{q}, \bar{y})<0$ to the strict inequality $H_{i^{\prime}}(\bar{q}, \tilde{y})<0$ at any of the critical neighbors $i^{\prime}$ of $i^{*}$ just by modifying the flow vector. This procedure can now be repeated for any of the critical neighbors of $i^{\prime}$, and so after finitely many steps one arrives at a feasible solution $\left(\bar{q}, y^{\prime}\right)$ of $(3.3)$ such that $H_{i^{* *}}\left(\bar{q}, y^{\prime}\right)<0$ for the node $i^{* *} \in \Pi$ constructed above. Since the objective function of (3.3) does not 
depend on $y$ but just on $q$, it follows from the fact that that $(\bar{q}, \bar{y})$ was an optimal solution to (3.3), that $\left(\bar{q}, y^{\prime}\right)$ is also an optimal solution to (3.3). But now, using the already proven fact that demand satisfaction comes as an equality at solutions to (3.3) for all active generators, we infer from the relation $i^{* *} \leq l$ shown above that $H_{i^{* *}}\left(\bar{q}, y^{\prime}\right)=0$, a contradiction. Consequently, our original assumption $H_{i^{*}}(\bar{q}, \bar{y})<0$ is wrong showing that $H_{i}(\bar{q}, \bar{y})=0$ for $i \in\{l+1, \ldots, N\}$. This completes the proof of the lemma.

The surjectivity condition in statement 1.(c) of Lemma 4.2 can be interpreted as follows in a special case: if $l=N-1$ (all generators but one are active) then there must exist at least one non-congested transmission line leading to the non-active generator.

We next turn to strong regularity of solutions to the ISO problem. We recall that the ISO problem (3.3) with fixed parameters $(\bar{\alpha}, \bar{\beta})$ satisfies the strong second-order sufficient condition (SSOSC) at one of its solutions $(\bar{q}, \bar{y})$ if

$$
\left\langle d, \nabla_{(q, y)}^{2} \mathcal{L}(\bar{q}, \bar{y}, \bar{\xi}) d\right\rangle>0 \quad \forall d \neq 0: \bar{\xi}_{i} \nabla H_{i}(\bar{q}, \bar{y}) d=0 \quad(i=1, \ldots, 2 N+m-l-k)
$$

holds for all $\bar{\xi}$ such that $\nabla_{(q, y)} \mathcal{L}(\bar{q}, \bar{y}, \bar{\xi})=0$. Here,

$$
\mathcal{L}(q, y, \xi):=\sum_{i=1}^{N} c_{i}\left(\bar{\alpha}_{i}, \bar{\beta}_{i}, q_{i}\right)+\sum_{i=1}^{2 N+m-l-k} \xi_{i} H_{i}(q, y)
$$

refers to the Lagrangian associated with (3.3) for fixed $(\alpha, \beta)=(\bar{\alpha}, \bar{\beta})$ and the $\bar{\xi}$ denote the Lagrange multipliers associated with the solution $(\bar{q}, \bar{y})$, assuming, of course, that they exist. Note that in the formulation of (4.17) we have already exploited the fact that all $2 N+m-l-k$ components of $H$ are active at $(\bar{q}, \bar{y})$ by statement 2 . of Lemma 4.2.

Proposition 4.1. Let $(\bar{\alpha}, \bar{\beta}, \bar{q}, \bar{y})$ be a solution to (3.5) satisfying (3.6). Then, there exists some $\Delta>0$ such that under the condition $\rho_{j} \in(0, \Delta)(j=1, \ldots, m)$ the following holds true: if $l=N$ (all generators active) or $k=m$ (no congestion) then $\nabla H(\bar{q}, \bar{y})$ is surjective and (4.17) is satisfied at $(\bar{q}, \bar{y})$. The same conclusion can also be drawn if $\rho_{j}=0(j=1, \ldots, m)$ under the additional assumption that the network graph is a tree (i.e., it does not contain cycles).

Proof. First note that $\nabla H(\bar{q}, \bar{y})$ is surjective under the assumption $l=N$ or $k=m$ by statement 1.(b) of Lemma 4.2 . Hence, the Lagrange multipliers are unique and it suffices to verify (4.17). Exploiting the explicit definitions of $c_{i}$ and $H$, one calculates

$$
\nabla_{(q, y)}^{2} \mathcal{L}(\bar{q}, \bar{y}, \bar{\xi})=\left(\begin{array}{cccc}
2[\operatorname{diag} \bar{\beta}] & 0 & \cdots & 0 \\
0 & \rho_{1} \sum_{i=1}^{N} \bar{\xi}_{i}\left|b_{i 1}\right| & \cdots & 0 \\
\vdots & \vdots & \ddots & \vdots \\
0 & 0 & \cdots & \rho_{m} \sum_{i=1}^{N} \bar{\xi}_{i}\left|b_{i m}\right|
\end{array}\right) .
$$

This diagonal matrix contains only positive entries. Indeed, this is clear for the first block because $\bar{\beta}_{i}>0$ (see (3.6)). For the remaining entries

$$
\rho_{j} \sum_{i=1}^{N} \bar{\xi}_{i}\left|b_{i j}\right|
$$

note that $\rho_{j}>0$ by assumption. Moreover, $\bar{\xi}_{i}>0$ for $i=1, \ldots, N$. To see this, recall that $\left(\bar{\xi}_{1}, \ldots, \bar{\xi}_{l}\right)$ and $\left(\bar{\xi}_{l+1}, \ldots, \bar{\xi}_{N}\right)$ correspond to the Lagrange multipliers of the demand satisfaction relations (first $N$ components of $H$ ) for active and non-active generators, respectively. Now statements 3.(a) and 3.(b) of Lemma 4.2 yield that strict complementarity (i.e., $\bar{\xi}_{i}>0$ for $i=1, \ldots, N$ ) holds whenever $l=N$ or $k=m$. On the other hand, as our network was supposed to be a connected graph, for each index $j$ there exists at least one (exactly: two) $i$ such that $\left|b_{i j}\right|=1$. Consequently, (4.18) is strictly positive for all $j=1, \ldots, m$. Now, with $\nabla_{(q, y)}^{2} \mathcal{L}(\bar{q}, \bar{y}, \bar{\xi})$ being positive definite, (4.17) is evidently satisfied at $(\bar{q}, \bar{y})$. 
For the second assertion, assume that $\rho_{j}=0(j=1, \ldots, m)$ and the network does not contain cycles. Then,

$$
\nabla_{(q, y)}^{2} \mathcal{L}(\bar{q}, \bar{y}, \bar{\xi})=\left(\begin{array}{cc}
2[\operatorname{diag} \bar{\beta}] & 0 \\
0 & 0
\end{array}\right)
$$

Choose an arbitrary $d$ as indicated in (4.17). In particular, $d \neq 0$. Moreover, as already shown above, one has that $\bar{\xi}_{i}>0$ for $i=1, \ldots, N$ holds whenever $l=N$ or $k=m$. Consequently, by $(4.17), \nabla H_{i}(\bar{q}, \bar{y}) d=0$ for $i=1, \ldots, N$. Using the partition $d=\left(d_{1}, d_{2}\right)$, the concrete shape of $\nabla H(\bar{q}, \bar{y})$ (see first two row blocks in (4.3) and notice that the part associated with the loss function vanishes due to $\rho_{j}=0$ for $j=1, \ldots, m$ ) yields that $d_{1}+B d_{2}=0$. However, since $B$ is injective as the incidence matrix of a tree, $d \neq 0$ already implies that $d_{1} \neq 0$. But then,

as was to be shown in (4.17).

$$
\left\langle d, \nabla_{(q, y)}^{2} \mathcal{L}(\bar{q}, \bar{y}, \bar{\xi}) d\right\rangle=2\left\langle d_{1},[\operatorname{diag} \bar{\beta}] d_{1}\right\rangle>0
$$

As a consequence of Proposition 4.1, the solutions to the ISO problem (3.3) are strongly regular in the sense of Robinson under the indicated assumptions, i.e., if $(\bar{\alpha}, \bar{\beta}, \bar{q}, \bar{y})$ is a solution to (3.5) satisfying (3.6), then, locally around $(\bar{\alpha}, \bar{\beta}, \bar{q}, \bar{y})$ all solutions of (3.3) can be parameterized by a single-valued and Lipschitzian mapping $(q(\alpha, \beta), y(\alpha, \beta))$. This allows, in particular, to locally replace the EPEC (3.5) by a simple equilibrium problem without explicit equilibrium constraints and thus to make it amenable to what is called the Implicit Programming Approach:

$$
\min _{\left(\alpha_{i}, \beta_{i}\right) \in \mathbb{R}^{2}}-f_{i}\left(\alpha_{i}, \beta_{i}, q(\alpha, \beta), y(\alpha, \beta)\right) \quad(i=1, \ldots, N)
$$

Note, however, that this approach is not possible in all relevant cases. For instance, if the network graph contains cycles, as it is typically the case, then Proposition 4.1 does not apply to the loss-free model $\left(\rho_{j}=0\right.$ $(j=1, \ldots, m))$.

\section{Stationarity of EPEC solutions}

Given the previous results pertaining to the structural properties of the spot market EPEC, we are now in a position to begin our analysis of stationary solutions to EPECs. Here, we base our approach on using so-called M-stationarity; meaning that the considered stationarity conditions are obtained on the basis of the limiting normal cone (see Sect. 2). We rely on a version of the M-stationary conditions for solutions to EPECs provided in [21] by Outrata which is based on a corresponding result for MPECs by Ye and Ye [28]. We adopt the statement to the setting of our spot market EPEC:

Theorem 5.1. Let $(\bar{\alpha}, \bar{\beta}, \bar{q}, \bar{y})$ be a solution to $(3.5)$. If for all $i=1, \ldots, N$, the multifunctions

$$
\Psi_{i}(u):=\left\{\left(\alpha_{i}, \beta_{i}, q, y\right) \mid u \in F\left(\bar{\alpha}_{-i}, \alpha_{i}, \bar{\beta}_{-i}, \beta_{i}, q, y\right)+N_{G}(q, y)\right\}
$$

are calm at $\left(0, \bar{\alpha}_{i}, \bar{\beta}_{i}, \bar{q}, \bar{y}\right)$ (see introduction), then for all $i=1, \ldots, N$, there exist $v^{i}$ such that the following relations hold

$$
\begin{aligned}
\nabla_{\left(\alpha_{i}, \beta_{i}\right)} f_{i}\left(\bar{\alpha}_{i}, \bar{\beta}_{i}, \bar{q}, \bar{y}\right) & =\nabla_{\left(\alpha_{i}, \beta_{i}\right)}^{T} F(\bar{\alpha}, \bar{\beta}, \bar{q}, \bar{y}) v^{i} \\
\nabla_{(q, y)} f_{i}\left(\bar{\alpha}_{i}, \bar{\beta}_{i}, \bar{q}, \bar{y}\right) & \in \nabla_{(q, y)}^{T} F(\bar{\alpha}, \bar{\beta}, \bar{q}, \bar{y}) v^{i}+D^{*} N_{G}(\bar{q}, \bar{y},-F(\bar{\alpha}, \bar{\beta}, \bar{q}, \bar{y}))\left(v^{i}\right) .
\end{aligned}
$$

Here, $D^{*}$ refers to the coderivative presented in the introduction. Before benefiting from this characterization of EPEC solutions one has to cope with two tasks: first, one has to be able to check the constraint qualification represented by the calmness of (5.1); second, an explicit formula for calculating the coderivative in terms of the problem data is needed. We address these tasks in the following two subsections. 


\subsection{Verification of calmness}

According to a well-known result by Robinson, a multifunction with polyhedral graph (i.e., the graph of which is a finite union of polyhedra) is calm at any point of its graph. Hence, the simplest way to verify calmness of (5.1) consists in checking the polyhedrality of its graph. The following observation is evident: if the mapping $F$ is linear and transmission losses are ignored, i.e., $\rho_{j}=0(j=1, \ldots, m)$, then the mappings $\Psi_{i}$ are calm at all points of their graph. Indeed, in the loss-free case the feasible set $G$ of the ISO problem (3.3) becomes a polyhedron, thus making the graph of the mapping $N_{G}$ a finite union of polyhedra. Then given the assumed linearity of $F$, the graph of $\Psi_{i}$ is also a finite union of polyhedra. Unfortunately, the mappings

$$
F\left(\bar{\alpha}_{-i}, \alpha_{i}, \bar{\beta}_{-i}, \beta_{i}, q, y\right)=\left(\begin{array}{c}
\left(\bar{\alpha}_{-i}, \alpha_{i}\right)+2\left[\operatorname{diag}\left(\bar{\beta}_{-i}, \beta_{i}\right)\right] q \\
0
\end{array}\right)
$$

are not linear in our case due to the bilinear term $\beta_{i} q_{i}$. Under the special assumption known as partial bidding ( $c f .[11,12])$, the producers' quadratic cost term is assumed to be known to every market participant and thus, it is not considered to be a the decision variable. In such a case, $F$ is indeed linear and the calmness of (5.1) can be taken for granted in the loss-free case (this fact was exploited, for instance in [7]). However here, we are not insisting on the rather restrictive assumption of partial bidding.

When polyhedrality fails, another way of verifying calmness is to check the stronger Aubin property (see introduction) by using available criteria. Indeed, we have the following result:

Proposition 5.1. Under the conditions of Proposition 4.1, the multifunctions $\Psi_{i}$ in (5.1) have the Aubin property and, hence, are calm at $\left(0, \bar{\alpha}_{i}, \bar{\beta}_{i}, \bar{q}, \bar{y}\right)$.

Proof. Under the assumptions of Proposition 4.1, surjectivity of $\nabla H(\bar{q}, \bar{y})$ holds true and the strong secondorder sufficient condition (4.17) is satisfied. By Theorem 4.1 in [24], the generalized equation

$$
0 \in \mathcal{C}(\alpha, \beta, q, y, \lambda)+N_{\mathbb{R}^{N+m} \times \mathbb{R}_{+}^{2 N+m-l-k}}(q, y, \lambda)
$$

with

$$
\mathcal{C}(\alpha, \beta, q, y, \lambda):=\left(\begin{array}{c}
F(\alpha, \beta, q, y)+\nabla^{T} H(q, y) \lambda \\
-H(q, y)
\end{array}\right)
$$

is strongly regular (see introduction) at $(\bar{\alpha}, \bar{\beta}, \bar{q}, \bar{y}, \bar{\lambda})$, where $\bar{\lambda}$ is the uniquely defined (by surjectivity of $\nabla H(\bar{q}, \bar{y})$ ) Lagrange multiplier satisfying $\nabla^{T} H(\bar{q}, \bar{y}) \bar{\lambda}=-F(\bar{\alpha}, \bar{\beta}, \bar{q}, \bar{y})$. Consequently, the mapping $\mathcal{F}$ defined by the partial linearization of $\mathcal{C}$ as

$$
\begin{aligned}
\mathcal{F}(\eta):= & \left\{(q, y, \lambda) \mid \eta \in \mathcal{C}(\bar{\alpha}, \bar{\beta}, \bar{q}, \bar{y}, \bar{\lambda})+\nabla_{(q, y, \lambda)} \mathcal{C}(\bar{\alpha}, \bar{\beta}, \bar{q}, \bar{y}, \bar{\lambda})((q, y, \lambda)-(\bar{q}, \bar{y}, \bar{\lambda}))\right. \\
& \left.+N_{\mathbb{R}^{N+m} \times \mathbb{R}_{+}^{2 N+m-l-k}}(q, y, \lambda)\right\}
\end{aligned}
$$

is locally Lipschitz and single-valued around $(0, \bar{q}, \bar{y}, \bar{\lambda})$ (see introduction). Next we put

$$
\Phi(\xi, q, y, \lambda):=\left(\begin{array}{c}
F(\bar{\alpha}, \bar{\beta}, \bar{q}, \bar{y})+\nabla_{(q, y)} F(\bar{\alpha}, \bar{\beta}, \bar{q}, \bar{y})((q, y)-(\bar{q}, \bar{y}))+\nabla^{T} H(q, y) \lambda \\
-H(q, y)
\end{array}\right)-\xi .
$$

It is immediately seen that

$$
\mathcal{C}(\bar{\alpha}, \bar{\beta}, \bar{q}, \bar{y}, \bar{\lambda})=\Phi(0, \bar{q}, \bar{y}, \bar{\lambda}), \quad \nabla_{(q, y, \lambda)} \mathcal{C}(\bar{\alpha}, \bar{\beta}, \bar{q}, \bar{y}, \bar{\lambda})=\nabla_{(q, y, \lambda)} \Phi(0, \bar{q}, \bar{y}, \bar{\lambda}) .
$$

Consequently,

$$
\begin{aligned}
\mathcal{F}(\eta)= & \left\{(q, y, \lambda) \mid \eta \in \Phi(0, \bar{q}, \bar{y}, \bar{\lambda})+\nabla_{(q, y, \lambda)} \Phi(0, \bar{q}, \bar{y}, \bar{\lambda})((q, y, \lambda)-(\bar{q}, \bar{y}, \bar{\lambda}))\right. \\
& \left.+N_{\mathbb{R}^{N+m} \times \mathbb{R}_{+}^{2 N+m-l-k}}(q, y, \lambda)\right\} .
\end{aligned}
$$


Since $\mathcal{F}$ is locally Lipschitz and single-valued around $(0, \bar{q}, \bar{y}, \bar{\lambda})$, it follows that the generalized equation

$$
0 \in \Phi(\xi, q, y, \lambda)+N_{\mathbb{R}^{N+m} \times \mathbb{R}_{+}^{2 N+m-l-k}}(q, y, \lambda)
$$

is strongly regular at $(0, \bar{q}, \bar{y}, \bar{\lambda})$ and, hence (see introduction), that the mapping

$$
\xi \mapsto\left\{(q, y, \lambda) \mid 0 \in \Phi(\xi, q, y, \lambda)+N_{\mathbb{R}^{N+m} \times \mathbb{R}_{+}^{2 N+m-l-k}}(q, y, \lambda)\right\}
$$

is locally Lipschitz and single-valued around $(0, \bar{q}, \bar{y}, \bar{\lambda})$. By definition of $\Phi$ and upon observing that

$$
N_{\mathbb{R}^{N+m} \times \mathbb{R}_{+}^{2 N+m-l-k}}(q, y, \lambda)=\{0\} \times N_{\mathbb{R}_{+}^{2 N+m-l-k}}(\lambda)
$$

this amounts to saying that the mapping

$$
\begin{aligned}
\xi \mapsto & \left\{(q, y, \lambda) \mid\left(\begin{array}{c}
\xi_{1} \\
\xi_{2}
\end{array}\right)\right. \\
& \left.\in\left(\begin{array}{c}
F(\bar{\alpha}, \bar{\beta}, \bar{q}, \bar{y})+\nabla_{(q, y)} F(\bar{\alpha}, \bar{\beta}, \bar{q}, \bar{y})((q, y)-(\bar{q}, \bar{y}))+\nabla^{T} H(q, y) \lambda \\
-H(q, y)+N_{\mathbb{R}_{+}^{2 N+m-l-k}}(\lambda)
\end{array}\right)\right\}
\end{aligned}
$$

is locally Lipschitz and single-valued around $(0, \bar{q}, \bar{y}, \bar{\lambda})$. Consequently, the mapping

$$
\begin{aligned}
\xi_{1} \mapsto & \left\{(q, y, \lambda) \mid\left(\begin{array}{c}
\xi_{1} \\
0
\end{array}\right)\right. \\
& \left.\in\left(\begin{array}{c}
F(\bar{\alpha}, \bar{\beta}, \bar{q}, \bar{y})+\nabla_{(q, y)} F(\bar{\alpha}, \bar{\beta}, \bar{q}, \bar{y})((q, y)-(\bar{q}, \bar{y}))+\nabla^{T} H(q, y) \lambda \\
-H(q, y)+N_{\mathbb{R}_{+}^{2 N+m-l-k}}(\lambda)
\end{array}\right)\right\}
\end{aligned}
$$

is locally Lipschitz and single-valued around $(0, \bar{q}, \bar{y}, \bar{\lambda})$. This implies that also the mapping

$$
\xi_{1} \mapsto\left\{(q, y) \mid \xi_{1} \in F(\bar{\alpha}, \bar{\beta}, \bar{q}, \bar{y})+\nabla_{(q, y)} F(\bar{\alpha}, \bar{\beta}, \bar{q}, \bar{y})((q, y)-(\bar{q}, \bar{y}))+N_{G}(q, y)\right\}
$$

is locally Lipschitz and single-valued around $(0, \bar{q}, \bar{y})$. Indeed, note first that in view of the surjectivity of $\nabla H(\bar{q}, \bar{y})$, one has

$$
N_{G}(q, y)=\left\{v \mid \exists \lambda: v=\nabla^{T} H(q, y) \lambda, H(q, y) \in N_{\mathbb{R}_{+}^{2 N+m-l-k}}(\lambda)\right\}
$$

for $(q, y)$ close to $(\bar{q}, \bar{y})$. Moreover, again by surjectivity of $\nabla H(\bar{q}, \bar{y})$ and due to $\nabla^{T} H(\bar{q}, \bar{y}) \bar{\lambda}=-F(\bar{\alpha}, \bar{\beta}, \bar{q}, \bar{y})$, the equation

$$
\xi_{1}-F(\bar{\alpha}, \bar{\beta}, \bar{q}, \bar{y})-\nabla_{(q, y)} F(\bar{\alpha}, \bar{\beta}, \bar{q}, \bar{y})((q, y)-(\bar{q}, \bar{y}))=\nabla^{T} H(q, y) \lambda
$$

has a unique solution $\lambda$ which is close to $\bar{\lambda}$ if $\left(\xi_{1}, q, y\right)$ is close to $(0, \bar{q}, \bar{y})$. These two observations allow to transfer the local Lipschitz and uniqueness statement from (5.4) to (5.5). This, however, means that the generalized equations

$$
0 \in F\left(\bar{\alpha}_{-i}, \alpha_{i}, \bar{\beta}_{-i}, \beta_{i}, q, y\right)+N_{G}(q, y) \quad(i=1, \ldots, N)
$$

are strongly regular at $\left(\bar{\alpha}_{i}, \bar{\beta}_{i}, \bar{q}, \bar{y}\right)$. Then, from Proposition 3.2 in [20], we infer that the mappings

$$
\left(u_{1}, u_{2}, u_{3}\right) \mapsto\left\{\left(\alpha_{i}, \beta_{i}, q, y\right) \mid u_{3} \in F\left(\bar{\alpha}_{-i}, \alpha_{i}, \bar{\beta}_{-i}, \beta_{i}, q, y\right)+N_{G}\left(u_{1}+q, u_{2}+y\right)\right\}
$$


have the Aubin property around $\left(0, \bar{\alpha}_{i}, \bar{\beta}_{i}, \bar{q}, \bar{y}\right)$. All the more, the restricted mappings

$$
\left(0,0, u_{3}\right) \mapsto\left\{\left(\alpha_{i}, \beta_{i}, q, y\right) \mid u_{3} \in F\left(\bar{\alpha}_{-i}, \alpha_{i}, \bar{\beta}_{-i}, \beta_{i}, q, y\right)+N_{G}(q, y)\right\},
$$

which are nothing else but $\Psi_{i}$, have the Aubin property around $\left(0, \bar{\alpha}_{i}, \bar{\beta}_{i}, \bar{q}, \bar{y}\right)$.

Note, that Proposition 5.1 cannot be applied in the loss-free case whenever the network graph contains cycles because then Proposition 4.1 cannot be invoked. Indeed, the following example demonstrates that the Aubin property for the mappings $\Psi_{i}$ in (5.1) gets lost in such cases.

Example 5.1. Given the spot market EPEC (3.5), let $(\bar{\alpha}, \bar{\beta}, \bar{q}, \bar{y})$ be a solution such that (3.6) is satisfied. Furthermore, let $N=3, m=3, l=3, k=3, \rho_{j}=0(j=1,2,3)$, i.e., there is neither congestion nor transmission losses nor non-active generators, and define a cyclic graph via the incidence matrix

$$
B=\left(\begin{array}{ccc}
-1 & 0 & 1 \\
1 & -1 & 0 \\
0 & 1 & -1
\end{array}\right)
$$

Since $l=N$, Lemma 4.2 indicates that $\nabla H(\bar{q}, \bar{y})$ is surjective. Then we can rewrite the normal cone $N_{G}(\bar{q}, \bar{y})$ (cf. Thm. $6.14[25]$ )

$$
N_{G}(\bar{q}, \bar{y})=\nabla^{T} H(\bar{q}, \bar{y}) N_{\mathbb{R}_{-}^{3}}(H(\bar{q}, \bar{y})) .
$$

From $\nabla H(\bar{q}, \bar{y})=(-I \mid-B)$ (see (4.3) with the special data of this example) and from the concrete shapes of $F$ and $B$ we derive that, for any $i \in\{1, \ldots, N\}$,

$$
\begin{aligned}
u & =\left(u_{1}, \ldots, u_{6}\right) \in F\left(\bar{\alpha}_{-i}, \alpha_{i}, \bar{\beta}_{-i}, \beta_{i}, q, y\right)+N_{G}(\bar{q}, \bar{y}) \Longrightarrow \\
\exists \bar{\lambda} & =\left(\bar{\lambda}_{1}, \bar{\lambda}_{2}, \bar{\lambda}_{3}\right) \in N_{\mathbb{R}_{-}^{3}}(H(\bar{q}, \bar{y})): u_{4}=\bar{\lambda}_{1}-\bar{\lambda}_{2}, u_{5}=\bar{\lambda}_{2}-\bar{\lambda}_{3}, u_{6}=\bar{\lambda}_{3}-\bar{\lambda}_{1} .
\end{aligned}
$$

Consequently, if $u \in F\left(\bar{\alpha}_{-i}, \alpha_{i}, \bar{\beta}_{-i}, \beta_{i}, q, y\right)+N_{G}(\bar{q}, \bar{y})$, then necessarily $u_{4}+u_{5}+u_{6}=0$. By contraposition, if $u_{4}+u_{5}+u_{6} \neq 0$ for some $u$, then necessarily $\Psi_{i}(u)=\emptyset$ for the multifunctions defined in (5.1). As one may now easily construct a sequence $u^{(n)} \rightarrow 0$ with $\Psi_{i}\left(u^{(n)}\right)=\emptyset$, it follows that $\Psi_{i}$ cannot have the Aubin property around $\left(0, \bar{\alpha}_{i}, \bar{\beta}_{i}, \bar{q}, \bar{y}\right)$.

It follows that in the loss-free case one can neither rely on a polyhedrality argument nor on a verification of the Aubin property in order to verify calmness. Fortunately, the loss of polyhedrality turns out to be weak enough to allow a direct derivation of the calmness of $\Psi_{i}$. In Section 7 we prove the calmness result in a slightly more general setting then needed for our purposes here. This leads to the next proposition.

Proposition 5.2. Let $(\bar{\alpha}, \bar{\beta}, \bar{q}, \bar{y})$ be a solution to (3.5) satisfying (3.6). If for all $j=1, \ldots, m, \rho_{j}=0$, then for all $i=1, \ldots, N$ the multifunctions $\Psi_{i}$ in (5.1) are calm at $\left(0, \bar{\alpha}_{i}, \bar{\beta}_{i}, \bar{q}, \bar{y}\right)$.

Proof. Being that $\Psi_{i}$ arises from the first order optimality conditions of the ISO problem, we can use Theorem 7.1 for such a task. Without loss of generality, let $i=1$, then

$$
F\left(\bar{\alpha}_{-1}, \alpha_{1}, \bar{\beta}_{-1}, \beta_{1}, q, y\right)=\left(\begin{array}{c}
\alpha_{1}+2 \beta_{1} q_{1} \\
\bar{\alpha}_{-1}+2\left[\operatorname{diag} \bar{\beta}_{-1}\right] q_{-1} \\
0
\end{array}\right) .
$$

Thus, $F\left(\bar{\alpha}_{-1}, \alpha_{1}, \bar{\beta}_{-1}, \beta_{1}, q, y\right)$ has the form:

$$
F\left(\bar{\alpha}_{-1}, \alpha_{1}, \bar{\beta}_{-1}, \beta_{1}, q, y\right)=\left(\begin{array}{c}
\Delta_{1}\left(\alpha_{1}, \beta_{1}, q, y\right) \\
\Delta_{2}(q, y)
\end{array}\right),
$$


where $\Delta_{1}\left(\alpha_{1}, \beta_{1}, q, y\right)=\alpha_{1}+2 \beta_{1} q_{1}$ and

$$
\Delta_{2}(q, y)=\left(\begin{array}{c}
\bar{\alpha}_{-1} \\
0
\end{array}\right)+\left(\begin{array}{ccc}
0 & 2\left[\operatorname{diag} \bar{\beta}_{-1}\right] & 0 \\
0 & 0 & 0
\end{array}\right)\left(\begin{array}{c}
q_{1} \\
q_{-1} \\
y
\end{array}\right)
$$

Then assumption 1. of Theorem 7.1 is fulfilled if we substitute $\left(\alpha_{1}, \beta_{1}\right)=u_{1},(q, y)=z, t_{1}=1, t_{2}=N-1+m$, and $C=G$. Moreover, $G$ is polyhedral and convex, as $H(q, y)$ is affine linear due to the fact that $\rho_{j}=0$ for all $j=1, \ldots, m$. Then Lemma 4.2 (statement 1.(a)) ensures the existence of a Slater point $(\tilde{q}, \tilde{y}) \in G$. Thus, assumption 2. is satisfied. Finally, by noting that

$$
\nabla_{\left(\alpha_{1}, \beta_{1}\right)} \Delta_{1}\left(\bar{\alpha}_{1}, \bar{\beta}_{1}, \bar{q}, \bar{y}\right)=\left(1,2 \bar{q}_{1}\right) \neq(0,0),
$$

we see that assumption 3 . is fulfilled. Therefore, Theorem 7.1 implies $\Psi_{1}$ is calm at $\left(0, \bar{\alpha}_{1}, \bar{\beta}_{1}, \bar{q}, \bar{y}\right)$.

\subsection{A transformation formula for the coderivative}

In this subsection we deal with the question of how to calculate the coderivative $D^{*} N_{G}$ in the stationarity conditions (5.3). Note that calculating this coderivative from scratch on the basis of the definition provided in the introduction may be very difficult, if at all possible. On the other hand, one has access to a simple formula for $D^{*} N_{\mathbb{R}_{-}^{p}}$ and may then use a convenient transformation formula to get $D^{*} N_{G}$ in the case where $G$ is described by finitely many smooth inequalities satisfying a constraint qualification. More precisely, we first have (see e.g., [5] or [19]): For any $(\tilde{z}, \tilde{v}) \in \operatorname{gr} N_{\mathbb{R}_{-}^{p}}$ it holds that for all $\tilde{v}^{*} \in \mathbb{R}^{p}$

$$
D^{*} N_{\mathbb{R}_{-}^{p}}(\tilde{z}, \tilde{v})\left(\tilde{v}^{*}\right)=\left\{\begin{array}{cl}
\emptyset & \text { if } \exists i: \tilde{v}_{i} \tilde{v}_{i}^{*} \neq 0 \\
\left\{x^{*} \in \mathbb{R}^{t} \mid x_{i}^{*}=0 \forall i \in I^{a}, x_{i}^{*} \geq 0 \forall i \in I^{b}\right\} & \text { else, }
\end{array}\right.
$$

where

$$
I^{a}:=\left\{i \mid \tilde{z}_{i}<0\right\} \cup\left\{i \mid \tilde{v}_{i}=0, \tilde{v}_{i}^{*}<0\right\}, \quad I^{b}:=\left\{i \mid \tilde{z}_{i}=0, \tilde{v}_{i}=0, \tilde{v}_{i}^{*}>0\right\} .
$$

Second, the following transformation formula was proved in [18] (Thm. 3.4), [17] (Thm. 1.127):

Theorem 5.2. Let $C:=\left\{z \in \mathbb{R}^{t} \mid A_{i}(z) \leq 0(i=1, \ldots, p)\right\}$, where $A \in \mathcal{C}^{2}\left(\mathbb{R}^{t} ; \mathbb{R}^{p}\right)$. Fix $\bar{z} \in C$ and $\bar{v} \in N_{C}(\bar{z})$, with $A(\bar{z})=0$. If the vectors $\left\{\nabla A_{i}(\bar{z})\right\}_{i=1}^{p}$ are linearly independent (i.e., $\nabla A(\bar{z})$ is surjective), then for all $v^{*} \in \mathbb{R}^{t}$

$$
D^{*} N_{C}(\bar{z}, \bar{v})\left(v^{*}\right)=\left(\sum_{i=1}^{p} \bar{\lambda}_{i} \nabla^{2} A_{i}(\bar{z})\right) v^{*}+\nabla^{T} A(\bar{z}) D^{*} N_{\mathbb{R}_{-}^{p}}(0, \bar{\lambda})\left(\nabla A(\bar{z}) v^{*}\right),
$$

where $\bar{\lambda}$ is uniquely defined by the relation $\nabla^{T} A(\bar{z}) \bar{\lambda}=\bar{v}$.

Evidently, (5.7) allows to calculate explicitly $D^{*} N_{C}$ upon using (5.6).

Concerning the second term occurring in both formulae (5.7) and (5.9), we derive from (5.6) that:

$$
\begin{gathered}
\nabla^{T} A(\bar{z}) D^{*} N_{\mathbb{R}_{-}^{p}}(0, \bar{\lambda})\left(\nabla A(\bar{z}) v^{*}\right)= \\
\emptyset \\
\left\{\begin{array}{cl}
\emptyset & \text { if } \exists i: \bar{\lambda}_{i} \nabla A_{i}(\bar{z}) v^{*} \neq 0 \\
\nabla^{T} A(\bar{z})\left\{x^{*} \in \mathbb{R}^{t} \mid x_{i}^{*}=0 \forall i \in I^{a}, \quad x_{i}^{*} \geq 0 \forall i \in I^{b}\right\} & \text { else, }
\end{array}\right.
\end{gathered}
$$

where

$$
I^{a}:=\left\{i \mid \bar{\lambda}_{i}=0, \nabla A_{i}(\bar{z}) v^{*}<0\right\}, \quad I^{b}:=\left\{i \mid \bar{\lambda}_{i}=0, \nabla A_{i}(\bar{z}) v^{*}>0\right\} .
$$


If $D^{*} N_{\mathbb{R}_{-}^{p}}(0, \bar{\lambda})\left(\nabla A(\bar{z}) v^{*}\right) \neq \emptyset$, then one arrives at the representation

$$
\nabla^{T} A(\bar{z}) D^{*} N_{\mathbb{R}_{-}^{p}}(0, \bar{\lambda})\left(\nabla A(\bar{z}) v^{*}\right)=\left\{\nabla^{T} A(\bar{z}) x^{*} \mid x_{i}^{*}=0\left(i \in I^{a}\right), x_{i}^{*} \geq 0\left(i \in I^{b}\right)\right\} .
$$

Moreover, the assumed non-emptiness implies the relation

$$
\nabla A_{i}(\bar{z}) v^{*}=0 \quad \forall i: \bar{\lambda}_{i}>0 .
$$

Now we are in a position to reformulate the abstract stationarity conditions of Theorem 5.1 in terms of our concrete EPEC. We provide the result for two significant constellations:

Theorem 5.3. Let $(\bar{\alpha}, \bar{\beta}, \bar{q}, \bar{y})$ be a solution to (3.5) satisfying (3.6). Assume that $l=N$ (all generators active) or $k=m$ (no congestion). Then there exists some $\Delta>0$ such that under the condition $\rho_{j} \in(0, \Delta)$ $(j=1, \ldots, m)$ (small positive losses) or $\rho_{j}=0(j=1, \ldots, m)$ (no losses), the following holds true: there exists some $\bar{\lambda} \in \mathbb{R}_{+}^{2 N+m-l-k}$ and for all $i=1, \ldots, N$, there exist $\left(v^{i}, w^{i}\right) \in \mathbb{R}^{N+m} \times \mathbb{R}^{2 N+m-l-k}$ such that

$$
\begin{aligned}
\nabla_{\left(\alpha_{i}, \beta_{i}\right)} f_{i}\left(\bar{\alpha}_{i}, \bar{\beta}_{i}, \bar{q}, \bar{y}\right) & =\nabla_{\left(\alpha_{i}, \beta_{i}\right)}^{T} F(\bar{\alpha}, \bar{\beta}, \bar{q}, \bar{y}) v^{i} \\
\nabla_{(q, y)} f_{i}\left(\bar{\alpha}_{i}, \bar{\beta}_{i}, \bar{q}, \bar{y}\right) & =\nabla_{(q, y)}^{T} F(\bar{\alpha}, \bar{\beta}, \bar{q}, \bar{y}) v^{i}+\left(\sum_{j=1}^{N} \bar{\lambda}_{j} \nabla^{2} H_{j}(\bar{q}, \bar{y})\right) v^{i}+\nabla^{T} H(\bar{q}, \bar{y}) w^{i} \\
\nabla H_{j}(\bar{q}, \bar{y})^{i} & =0 \quad \forall j: \bar{\lambda}_{j}>0 \\
\left(w^{i}\right)_{j} & =0 \quad \forall j: \bar{\lambda}_{j}=0, \nabla H_{j}(\bar{q}, \bar{y}) v^{i}<0 \\
\left(w^{i}\right)_{j} & \geq 0 \quad \forall j: \bar{\lambda}_{j}=0, \nabla H_{j}(\bar{q}, \bar{y}) v^{i}>0 \\
F(\bar{\alpha}, \bar{\beta}, \bar{q}, \bar{y}) & =-\nabla^{T} H(\bar{q}, \bar{y}) \bar{\lambda} .
\end{aligned}
$$

Proof. Note first that in all indicated constellations, $\nabla H(\bar{q}, \bar{y})$ is surjective according to Lemma 4.2, statement 1.(b) and that the calmness assumption for the multifunctions (5.1) is satisfied by virtue of Propositions 5.1 (for the case $\rho_{j} \in(0, \Delta)$ ) and 5.2 (for the case $\rho_{j}=0$ ). Thus, we may apply Theorem 5.1. (5.2) immediately yields (5.11). Given the surjectivity of $\nabla H(\bar{q}, \bar{y})$, we may apply Theorem 5.2 in order to derive (5.12) from (5.3) by combining (5.7) with (5.9) and putting $G:=C, H:=A$ and $p:=2 N+m-l-k$. Note that in the summation of Hessian terms in (5.12) one may omit indices $j>N$ due to the components $H_{j}$ being linear then. (5.13) follows from (5.10). (5.14) and (5.15) follow from the definitions of the index sets $I^{a}$ and $I^{b}$ introduced in (5.8). Finally, (5.16) characterizes the uniquely determined (by surjectivity of $\nabla H(\bar{q}, \bar{y})$ ) multiplier $\bar{\lambda}$ from the solution of the ISO problem (3.3).

The assumptions of Theorem 5.3 guarantee that $\nabla H_{j}(\bar{q}, \bar{y})$ is surjective by Lemma 4.2, statement 1.(b). If, however, both congestion and inactivity takes place at an EPEC solution (i.e., $l<N$ and $k<m$ ), then surjectivity of $\nabla H(\bar{q}, \bar{y})$ may be lost. An example is given by $N=3$ producers the first $l=2$ of which are active and connected by a line without congestion, whereas the third producer is inactive and connected with the second one via a congested line (hence, $k=1$ ). On the other hand, we know by Lemma 4.2, statement 1.(a) that at least positive linear independence of the rows of $\nabla H(\bar{q}, \bar{y})$ remains valid. In such a case the transformation formula (5.7) was found to still hold true as an inclusion " $\subseteq$ ", where on the right-hand side of (5.7) the union over the whole set of admissible Lagrange multipliers has to be taken (see [19], Thm. 3.1). This leads to a certain loss of information but still allows to determine stationarity conditions that EPEC solutions have to satisfy. 
Fortunately, in the linear case one is much better off as there still exists an exact coderivative formula even if $\nabla H(\bar{q}, \bar{y})$ is not surjective. More precisely, the following holds true (see [8], Thm. 3.2, and [7], Prop. 3.2):

Theorem 5.4. Let $C:=\left\{z \in \mathbb{R}^{t} \mid A z \leq b\right\}$, where $b \in \mathbb{R}^{p}$ and $A$ is a matrix of order $(p, t)$. Fix $\bar{z} \in C$ and $\bar{v} \in N_{C}(\bar{z})$ with $A \bar{z}=b$. Let $\bar{\lambda}$ be any vector defined by the relation $A \bar{\lambda}=\bar{v}$ (the existence of $\bar{\lambda}$ is guaranteed by $\bar{v} \in N_{C}(\bar{z})$ and $C$ being polyhedral). Denote

$$
J:=\left\{j \in\{1, \ldots, p\} \mid \lambda_{j}>0\right\} .
$$

Then,

$$
D^{*} N_{C}(\bar{z}, \bar{v})\left(v^{*}\right)=\left\{x^{*} \mid\left(x^{*},-v^{*}\right) \in \bigcup_{J \subseteq I_{1} \subseteq I_{2} \subseteq\{1, \ldots, p\}} P_{I_{1}, I_{2}} \times Q_{I_{1}, I_{2}}\right\},
$$

where

$$
\begin{aligned}
P_{I_{1}, I_{2}} & =\operatorname{con}\left\{a_{i} \mid i \in \chi\left(I_{2}\right) \backslash I_{1}\right\}+\operatorname{span}\left\{a_{i} \mid i \in I_{1}\right\} \\
Q_{I_{1}, I_{2}} & =\left\{h \in \mathbb{R}^{n} \mid\left\langle a_{i}, h\right\rangle=0\left(i \in I_{1}\right),\left\langle a_{i}, h\right\rangle \leq 0 \quad\left(i \in \chi\left(I_{2}\right) \backslash I_{1}\right)\right\},
\end{aligned}
$$

"con" and "span" refer to the conic convex and linear hulls, respectively, and

$$
\begin{aligned}
\chi\left(I^{\prime}\right):= & \left\{j \in\{1, \ldots, p\} \mid \text { if }\left\langle a_{i}, h\right\rangle \leq 0 \text { for } i \in\{1, \ldots, p\} \backslash I^{\prime} \quad\left(I^{\prime} \subseteq\{1, \ldots, p\}\right) .\right. \\
& \text { and } \left.\left\langle a_{i}, h\right\rangle=0 \text { for } i \in I^{\prime}, \text { then }\left\langle a_{j}, h\right\rangle=0\right\}
\end{aligned}
$$

Using the last theorem, one can derive M-stationarity conditions similar to those stated in Theorem 5.3 that would apply to settings where both congestion and inactivity occur simultaneously but losses are absent. We skip an explicit statement of these conditions here for the sake of brevity. We also note that Theorem 5.4 can be generalized to the case of finitely many linear inequalities in a reflexive Banach space (see [9], Thm. 4.1).

\section{Explicit CHARACTERIZATIONS OF SOLUtions TO THE SPOT MARKET EPEC USING M-STATIONARITY CONDITIONS}

With the results of the previous sections in mind, we now provide explicit characterizations of solutions to the spot market EPEC (3.5) via M-stationarity conditions. For the sake of brevity, we restrict ourselves to one particular solution setting, namely the loss-free case without congestion and with an acyclic network graph. In the absence of losses and congestion, the Jacobian of the mapping $H$ takes the form (see (4.3))

$$
\nabla H(\bar{q}, \bar{y})=\left(\begin{array}{ccc}
-I_{1} & 0 & -B^{1} \\
0 & -I_{2} & -B^{2} \\
0 & -I_{2} & 0
\end{array}\right)
$$

This leads to the next theorem.

Theorem 6.1. Let $(\bar{\alpha}, \bar{\beta}, \bar{q}, \bar{y})$ be a solution to (3.5) satisfying (3.6). Assume that $\rho_{j}=0$ for $j=1, \ldots, m$ (no losses) and $k=m$ (no congestion). Then there exists some $\bar{\lambda} \in \mathbb{R}_{+}^{2 N-l}$ and for all $i=1, \ldots, N$, there exist 
$\left(v^{i}, w^{i}\right) \in \mathbb{R}^{N+m} \times \mathbb{R}^{2 N-l}$ such that

$$
\begin{aligned}
\left(v^{i}\right)_{i} & =\left\{\begin{array}{c}
\bar{q}_{i} \quad \text { if } i \in\{1, \ldots, l\} \\
0 \quad \text { if } i \in\{l+1, \ldots, N\}
\end{array}\right. \\
v_{a}^{i}+B^{1} v_{c}^{i} & =v_{b}^{i}+B^{2} v_{c}^{i}=0 \\
\left(v^{i}\right)_{l+j} & =0 \quad \forall j \in\{1, \ldots, N-l\}: \bar{\lambda}_{N+j}>0 \\
\left(w^{i}\right)_{i} & =\gamma_{i}-\bar{\alpha}_{i}+2\left(\delta_{i}-\bar{\beta}_{i}\right) \bar{q}_{i} \quad \text { if } i \in\{1, \ldots, l\} \\
\left(w^{i}\right)_{i}+\left(w^{i}\right)_{N+i-l} & =\gamma_{i}-\bar{\alpha}_{i} \quad \text { if } i \in\{l+1, \ldots, N\} \\
\left(w^{i}\right)_{j} & =2 \bar{\beta}_{j}\left(v^{i}\right)_{j} \quad \text { if } j \in\{1, \ldots, l\}, j \neq i \\
\left(w^{i}\right)_{j}+\left(w^{i}\right)_{N+j-l} & =2 \bar{\beta}_{j}\left(v^{i}\right)_{j} \quad \text { if } j \in\{l+1, \ldots, N\}, j \neq i \\
\left(w^{i}\right)_{N+j-l} & =0 \quad \text { if } j \in\{l+1, \ldots, N\}, \bar{\lambda}_{N+j-l}=0,\left(v^{i}\right)_{j}>0 \\
\left(w^{i}\right)_{N+j-l} & \geq 0 \quad \text { if } j \in\{l+1, \ldots, N\}, \bar{\lambda}_{N+j-l}=0,\left(v^{i}\right)_{j}<0 \\
\left(B^{1}\right)^{T} w_{a}^{i}+\left(B^{2}\right)^{T} w_{b}^{i} & =0 \quad \bar{\alpha}_{j}+2 \bar{\beta}_{j} \bar{q}_{j} \quad(j=1, \ldots, l) \\
\bar{\alpha}_{1}+2 \bar{\beta}_{1} \bar{q}_{1} & =\bar{\alpha}_{j^{\prime}}-\bar{\lambda}_{N+j^{\prime}-l} \quad\left(j^{\prime}=l+1, \ldots, N\right) .
\end{aligned}
$$

Here $(\cdot)_{j}$ identifies a concrete component of a vector, whereas lower indices " $a$ ", "b", "c" obey the partition of the Jacobian in (6.1) and its transpose, respectively.

Proof. (6.2) follows from (5.11) upon calculating $\nabla_{\left(\alpha_{i}, \beta_{i}\right)} f_{i}(\bar{\alpha}, \bar{\beta}, \bar{q}, \bar{y})$ and $\nabla_{\left(\alpha_{i}, \beta_{i}\right)}^{T} F(\bar{\alpha}, \bar{\beta}, \bar{q}, \bar{y})$ and recalling that $\bar{q}_{i}>0$ for $i \leq l$ and $\bar{q}_{i}=0$ for $i \in\{l+1, \ldots, N\}$. Next, observe that due to our assumption $k=m$, we know that, by statement 3.(b) of Lemma $4.2, \bar{\lambda}_{j}>0$ for $j=1, \ldots, N$. Therefore, (6.3) and (6.4) are implied by (5.13) taking into account the shape of the Jacobian in (6.1). Relations (6.5)-(6.8) and (6.11) are derived from (5.12) (with the Hessian term missing due to linearity as a consequence of the loss-free case) upon calculating $\nabla_{(q, y)} f_{i}(\bar{\alpha}, \bar{\beta}, \bar{q}, \bar{y})$ and $\nabla_{(q, y)}^{T} F(\bar{\alpha}, \bar{\beta}, \bar{q}, \bar{y})$ and comparing components in the light of the shape of the transposed Jacobian in (6.1). Relations (6.9) and (6.10) correspond to (5.14) and (5.15), where again $\bar{\lambda}_{j}>0$ for $j=1, \ldots, N$ and the shape of the third row block in (6.1) are exploited. Relations (6.12) and (6.13) result from the KKT conditions of the ISO problem (3.3) along the lines of relations (4.7), (4.8) and (4.9). Indeed, (4.9) implies, due to the absence of losses and congestion, that

$$
\left(\bar{\lambda}_{1}, \ldots, \bar{\lambda}_{N}\right)^{T} \in \operatorname{ker} B^{T}
$$

which in turn results in $\bar{\lambda}_{1}=\ldots=\bar{\lambda}_{N}$ by virtue of Lemma 4.1, statement 1. Now, (6.12) and (6.13) follow from (4.7) and (4.8) with an appropriate change of notation.

As an illustration, we determine the M-stationary points in an example with two settlements, i.e., the network graph is given by a single transmission line connecting two nodes. Moreover we assume absence of transmission losses and congestion. We are interested in EPEC solutions in which one of the two producers has become inactive. Accordingly, let $(\bar{\alpha}, \bar{\beta}, \bar{q}, \bar{y})$ be a solution to (3.5) satisfying (3.6) such that $N=2, k=m=1, l=1$ and $\rho=0$. Then,

$$
B=\left(\begin{array}{c}
-1 \\
1
\end{array}\right), \quad \nabla H(\bar{q}, \bar{y})=\left(\begin{array}{ccc}
-1 & 0 & -1 \\
0 & -1 & 1 \\
0 & -1 & 0
\end{array}\right)
$$

Putting $i:=1$, (6.2) and (6.3) provide $v^{1}=\left(\bar{q}_{1},-\bar{q}_{1},-\bar{q}_{1}\right)$. In particular, since $\bar{q}_{1}>0$ (due to $\left.l=1\right)$, (6.4) (with $j:=1$ ) leads to $\bar{\lambda}_{3}=0$, since $v_{2}^{1}=-\bar{q}_{1}<0$. Using this last fact along with $(6.13)\left(\right.$ with $\left.j^{\prime}:=2\right)$, we derive that

$$
\bar{\alpha}_{1}+2 \bar{\beta}_{1} \bar{q}_{1}=\bar{\alpha}_{2}-\bar{\lambda}_{3}=\bar{\alpha}_{2} .
$$


Next, (6.5) and (6.8) (with $j:=2$ ) lead to

$$
\left(w^{1}\right)_{1}=\gamma_{1}-\bar{\alpha}_{1}+2\left(\delta_{1}-\bar{\beta}_{1}\right) \bar{q}_{1} ; \quad\left(w^{1}\right)_{2}+\left(w^{1}\right)_{3}=2 \bar{\beta}_{2}\left(v^{1}\right)_{2}=-2 \bar{\beta}_{2} \bar{q}_{1}
$$

Finally, (6.11) along with $B^{1}=-1, B^{2}=1$ implies $\left(w^{1}\right)_{1}=w_{a}^{1}=w_{b}^{1}=\left(w^{1}\right)_{2}$. This combines with the two relations above to

$$
\gamma_{1}-\bar{\alpha}_{1}+2\left(\delta_{1}-\bar{\beta}_{1}\right) \bar{q}_{1}+\left(w^{1}\right)_{3}=-2 \bar{\beta}_{2} \bar{q}_{1}
$$

Now, by virtue of $\bar{\lambda}_{3}=0$ and $v_{2}^{1}<0,(6.10)$ (with $j:=2$ ) allows us to deduce that $\left(w^{1}\right)_{3} \geq 0$. Inserting this into the relation above and recalling (6.14), we obtain the following relations, which an EPEC solution must necessarily satisfy under the indicated conditions:

$$
\bar{\alpha}_{2}=\bar{\alpha}_{1}+2 \bar{\beta}_{1} \bar{q}_{1} ; \quad \gamma_{1}-\bar{\alpha}_{1}+2\left(\delta_{1}-\bar{\beta}_{1}+\bar{\beta}_{2}\right) \bar{q}_{1} \leq 0
$$

At this point one might wonder if an evaluation of the necessary conditions of Theorem 6.1 also for the second generator (i.e., for $i=2$ ) would add some additional information. The answer is negative in this simple example because in contrast to the derivation with $i=1$ it is not possible to gain a characterization for the multiplier $\left(w^{1}\right)_{3}$. This is due to the fact that $v^{2}=0$, which can be as easily calculated as $v^{1}$ above. But then, neither (6.10) nor (6.9) apply. We also mention that the stationarity conditions from Theorem 6.1 do not allow to uniquely identify EPEC solutions. This however, is not a weakness of these conditions but relies on the fact that the EPEC solutions in our problem are not isolated but rather form a continuous set (see also [12]).

As stated in the introduction, we also seek to provide a quantitative economic interpretation of our stationarity conditions in the two-settlement example. To this aim, we reduce the obtained relations to fixed quantities of the problem (total demand and true cost coefficients of the generators) independent of the bid coefficients. Recall first that $H(\bar{q}, \bar{y})=0$ according to Lemma 4.2, statement 2. Given the mapping $H$ in our small example, this amounts to the relations

$$
\bar{q}_{1}+\bar{y}=d_{1}, \quad \bar{q}_{2}-\bar{y}=d_{2}, \quad \bar{q}_{2}=0 .
$$

Denoting $d:=d_{1}+d_{2}$ the total demand, these relations yield that $\bar{q}_{1}=d$. Now, using this and substituting for $\bar{\alpha}_{1}$ in the first relation of (6.15), the second relation of (6.15) leads to the inequality

$$
\gamma_{1}-\bar{\alpha}_{2}+2\left(\delta_{1}+\bar{\beta}_{2}\right) d \leq 0
$$

In the last step we exploit the extra information $\bar{\alpha}_{2} \leq \gamma_{2}$ (the bid linear coefficient of generator 2 is not larger than its true linear cost coefficient) which can be obtained from deriving the so-called strong stationarity conditions for our EPEC in the small example. We note that strong stationarity leads to more precise conditions than M-stationarity, but needs a far more restrictive constraint qualification than the latter in order to provide necessary optimality conditions. Now by adding the indicated extra information to the last obtained inequality and noting that $\bar{\beta}_{2}, d>0$, we end up with the strict inequality

$$
\gamma_{1}+2 \delta_{1} d<\gamma_{2}
$$

This last relation means the following: In order to make the second generator disappear from the market in an equilibrium solution, its true linear cost coefficient has to be strictly larger than the true linear cost coefficient of the first generator by an amount of at least $2 \delta_{1} d$, where $\delta_{1}$ is the true quadratic cost coefficient of the first generator and $d$ is the total demand. This statement obviously contains much more information than the intuitively easy to guess relation

$$
\gamma_{1}+2 \delta_{1} d<\gamma_{2}+2 \delta_{2} d
$$

which is a consequence of the first one and which expresses that the marginal price of generator 2 is strictly larger than that of generator 1 . 
Remark 6.1. The explicit relations formulated here serve a number of purposes. In a small setting, as illustrated in the example above, one is able to manipulate the various equalities and inequalities in order to directly characterize solutions. In larger situations, one may also use the conditions in conjunction with an existing numerical method to verify stationarity of computed solution candidates. Finally, and perhaps the aspect of greatest interest, it is possible to reformulate the explicit conditions derived in Theorem 5.3 as a system of smooth and non-smooth equations amenable to non-smooth Newton methods (cf. e.g., [13]). Future work in this direction is already in progress.

\section{On the Calmness of the solution mapping of a CANONiCAlly-Perturbed GENERALIZED EQUATION}

In the main result of this section which is also the basis for the proof of Proposition 5.2 we rely on the results of the following lemma, which is an immediate consequence of Theorem $4.10 \mathrm{in}$ [17], i.e., the so-called Mordukhovich Criterion (cf. Thm. 9.40 [25]):

Lemma 7.1. Let $Z: \mathbb{R}^{n} \rightrightarrows \mathbb{R}^{m}$ be a multifunction defined by

$$
Z(x):=\left\{y \in \mathbb{R}^{m} \mid h(x, y)=0, y \in \Omega\right\},
$$

where $h: \mathbb{R}^{n} \times \mathbb{R}^{m} \rightarrow \mathbb{R}^{k}$ is a continuously differentiable mapping and $\Omega \subseteq \mathbb{R}^{m}$ is closed. Consider a point $(\bar{x}, \bar{y}) \in \operatorname{gph} Z$. If for all $\left(x^{*}, y^{*}, z^{*}\right) \in \mathbb{R}^{n} \times \mathbb{R}^{m} \times \mathbb{R}^{k}$ the implication

$$
\left.\begin{array}{l}
x^{*}=\nabla_{x}^{T} h(\bar{x}, \bar{y}) z^{*} \\
y^{*}=-\nabla_{y}^{T} h(\bar{x}, \bar{y}) z^{*} \\
y^{*} \in N_{\Omega}(\bar{y})
\end{array}\right\} \Longrightarrow x^{*}=0
$$

holds true, then $Z$ enjoys the Aubin property around $(\bar{x}, \bar{y})$.

In the following, we assume, as in the spot market EPEC, that mappings $\Psi_{i}$ given in (5.1) arise from the first order optimality conditions of some perturbed optimization problem. More specifically, we define:

$$
\min _{z}\left\{f\left(u_{1}, z\right)-u_{2}^{T} z \mid \mathcal{A} z+b \in \mathbb{R}_{-}^{p}\right\},
$$

where $z \in \mathbb{R}^{t}, u_{1} \in \mathbb{R}^{s}, u_{2} \in \mathbb{R}^{t}, \mathcal{A} \in \mathbb{R}^{p \times t}, b \in \mathbb{R}^{p}$, and assume $f$ is continuously differentiable in both the decision variable $z$ and the parameter $u_{1}$. Then the first-order optimality conditions of (7.2) may be written:

$$
0 \in \nabla_{z} f\left(u_{1}, z\right)-u_{2}+N_{C}(z)
$$

where $C:=\left\{z \in \mathbb{R}^{t} \mid \mathcal{A} z+b \in \mathbb{R}_{-}^{p}\right\}$. Then we define

$$
\Psi\left(u_{2}\right):=\left\{\left(u_{1}, z\right) \in \mathbb{R}^{s+t} \mid u_{2} \in \nabla_{z} f\left(u_{1}, z\right)+N_{C}(z)\right\} .
$$

Theorem 7.1. Let $\left(\overline{u_{1}}, 0, \bar{z},\right)$ be a solution to (7.3) and assume the following conditions hold:

1.

$$
\nabla_{z} f\left(u_{1}, z\right)=\left(\begin{array}{c}
\Delta_{1}\left(u_{1}, z\right) \\
\Delta_{2}(z)
\end{array}\right)
$$

where $\Delta_{1} \in \mathcal{C}^{1}\left(\mathbb{R}^{s+t} ; \mathbb{R}^{t_{1}}\right)$ and $\Delta_{2}(z):=D z+c$ with $D \in \mathbb{R}^{t_{2} \times t}$ such that $t_{1}+t_{2}=t$ and $c \in \mathbb{R}^{t_{2}}$.

2. There exists $\tilde{z}$ such that $\mathcal{A} \tilde{z}+b \in \operatorname{int} \mathbb{R}_{-}^{p}$, i.e., there exists a Slater point.

3. $\nabla_{u_{1}} \Delta_{1}\left(\bar{u}_{1}, \bar{z}\right)$ is surjective.

Then the multifunction $\Psi$ in $(7.4)$ is calm at $\left(0, \bar{u}_{1}, \bar{z}\right)$. 
Proof. We begin by defining the following multifunction:

$$
\Phi\left(p_{1}, p_{2}\right):=\left\{\begin{array}{l|ccc}
\left(u_{1}, z, \lambda\right) \in \mathbb{R}^{s+t+p} & \Theta_{1}\left(u_{1}, z, \lambda\right) & = & p_{1} \\
\Theta_{2}(z, \lambda) & = & p_{2} \\
\lambda & \in & N_{\mathbb{R}_{-}^{p}}(\mathcal{A} z+b)
\end{array}\right\}
$$

where

$$
\Theta_{1}\left(u_{1}, z, \lambda\right):=\Delta_{1}\left(u_{1}, z\right)+\mathcal{A}_{1}^{T} \lambda ; \quad \Theta_{2}(z, \lambda):=\Delta_{2}(z)+\mathcal{A}_{2}^{T} \lambda
$$

such that $\mathcal{A}_{1} \in \mathbb{R}^{p \times t_{1}}, \mathcal{A}_{2} \in \mathbb{R}^{p \times t_{2}}$, and $\mathcal{A}=\left(\mathcal{A}_{1} \mid \mathcal{A}_{2}\right)$. Since $C$ is polyhedral,

$$
N_{C}(z)=\mathcal{A}^{T} N_{\mathbb{R}_{-}^{p}}(\mathcal{A} z+b) .
$$

Consequently, by partitioning $u_{2}=\left(u_{2}^{a}, u_{2}^{b}\right) \in \mathbb{R}^{t_{1}} \times \mathbb{R}^{t_{2}}$, we have

$$
\Psi\left(u_{2}\right)=\left\{\left(u_{1}, z\right) \in \mathbb{R}^{s+t} \mid \exists \lambda:\left(u_{1}, z, \lambda\right) \in \Phi\left(u_{2}^{a}, u_{2}^{b}\right)\right\}
$$

We now demonstrate, for an arbitrarily fixed $\bar{\lambda}$ with $\left(0,0, \bar{u}_{1}, \bar{z}, \bar{\lambda}\right) \in \operatorname{gph} \Phi$, that $\Phi$ is calm at $\left(0,0, \bar{u}_{1}, \bar{z}, \bar{\lambda}\right)$, after which we show how this implies $\Psi$ is also calm at $\left(0, \bar{u}_{1}, \bar{z}\right)$. Start by realizing that $\Phi\left(p_{1}, p_{2}\right)$ can be written as $\Phi\left(p_{1}, p_{2}\right)=\mathcal{S}\left(p_{1}\right) \cap \mathcal{T}\left(p_{2}\right)$, where

$$
\begin{array}{llll}
\mathcal{S}\left(p_{1}\right):= & \left\{\left(u_{1}, z, \lambda\right) \mid \Theta_{1}\left(u_{1}, z, \lambda\right)\right. & \left.=p_{1}\right\} \\
\mathcal{T}\left(p_{2}\right):= & \left\{\left(u_{1}, z, \lambda\right) \mid \begin{array}{ccc}
\Theta_{2}(z, \lambda) & = & p_{2} \\
\lambda & \in & N_{\mathbb{R}_{-}^{p}}(\mathcal{A} z+b)
\end{array}\right\} .
\end{array}
$$

Moreover, note that

$$
\left(\bar{u}_{1}, \bar{z}, \bar{\lambda}\right) \in \Phi(0,0) \Rightarrow\left(\bar{u}_{1}, \bar{z}, \bar{\lambda}\right) \in \mathcal{S}(0) \cap \mathcal{T}(0) .
$$

Thus, we can show that $\Phi$ is calm at $\left(0,0, \bar{u}_{1}, \bar{z}, \bar{\lambda}\right)$ by using an intersection criterion developed by Klatte and Kummer (see [14], Thm. 3.6): If the following conditions holds, then $\Phi$ is calm at $\left(0,0, \bar{u}_{1}, \bar{z}, \bar{\lambda}\right)$ :

$$
\begin{aligned}
& \mathcal{S} \text { is calm at }\left(0, \bar{u}_{1}, \bar{z}, \bar{\lambda}\right) \\
& \mathcal{T} \text { is calm at }\left(0, \bar{u}_{1}, \bar{z}, \bar{\lambda}\right)
\end{aligned}
$$

$\mathcal{S}^{-1}$ has the Aubin property around $\left(\bar{u}_{1}, \bar{z}, \bar{\lambda}, 0\right)$

$$
\mathcal{S} \cap \mathcal{T}(0) \text { is calm at }\left(0, \bar{u}_{1}, \bar{z}, \bar{\lambda}\right) \text {. }
$$

Since the multifunction $\mathcal{T}$ is polyhedral, it follows from [23] that it is calm at $\left(0, \bar{u}_{1}, \bar{z}, \bar{\lambda}\right)$. Thus, we show the remaining three conditions hold true. This will be done by checking the partially strengthened (from calmness towards Aubin property) conditions:

1. $\mathcal{S}$ has the Aubin property around $\left(0, \bar{u}_{1}, \bar{z}, \bar{\lambda}\right)$.

2. $\mathcal{S} \cap \mathcal{T}(0)$ has the Aubin property around $\left(0, \bar{u}_{1}, \bar{z}, \bar{\lambda}\right)$.

3. $\mathcal{S}^{-1}$ has the Aubin property around $\left(\bar{u}_{1}, \bar{z}, \bar{\lambda}, 0\right)$.

Recall Lemma 7.1. For 1. and 2. put there $x:=p_{1}, y:=\left(u_{1}, z, \lambda\right), h(x, y):=\Theta_{1}(y)-x$, and $\Omega:=\mathbb{R}^{m}$ for 1 . and $\Omega:=\mathcal{T}(0)$ for 2 ., respectively. Then $Z=\mathcal{S}$ in 1 . and $Z=\mathcal{S} \cap \mathcal{T}(0)$ in 2. The first relation in the assumption of (7.1) then yields $x^{*}=-z^{*}$. Hence, the second relation amounts to $y^{*}=\nabla_{y}^{T} h(\bar{x}, \bar{y}) x^{*}$. By using the partition $y^{*}=\left(u_{1}^{*}, z^{*}, \lambda^{*}\right)$, the first component of the previous relation, in accordance with that of $y$, now reads

$$
u_{1}^{*}=\nabla_{u_{1}}^{T} h(\bar{x}, \bar{y}) x^{*}=\nabla_{u_{1}}^{T} \Theta_{1}\left(\bar{u}_{1}, \bar{z}, \bar{\lambda}\right) x^{*} .
$$


By observing that $\mathcal{T}(0)=\mathbb{R}^{s} \times \mathcal{T}^{\prime}$ for some $\mathcal{T}^{\prime} \subseteq \mathbb{R}^{t} \times \mathbb{R}^{p}$, we have in both cases 1 . and 2 . that $\Omega=\mathbb{R}^{s} \times \mathcal{T}^{\prime \prime}$ for some $\mathcal{T}^{\prime \prime} \subseteq \mathbb{R}^{t} \times \mathbb{R}^{p}$. Consequently, the third relation in the assumption (7.1) yields $u_{1}^{*}=0$ in either case and hence, upon using the explicit structure of $\Theta_{1},(7.1)$ reduces to

$$
\nabla_{u_{1}}^{T} \Delta_{1}\left(\bar{u}_{1}, \bar{z}\right) x^{*}=0 \Rightarrow x^{*}=0
$$

This of course follows immediately from assumption 3. of the current theorem. For the proof of 3 ., put $y:=p_{1}$, $x:=\left(u_{1}, z, \lambda\right), h(x, y):=\Theta_{1}(x)-y$, and $\Omega:=\mathbb{R}^{t_{1}}$. Then $Z=S^{-1}$ and the assumption of (7.1) trivially imply $x^{*}=0$.

Since $\bar{\lambda}$ was chosen arbitrarily, we have shown that $\Phi$ is calm at $\left(0,0, \bar{u}_{1}, \bar{z}, \lambda\right)$ for any $\lambda$ such that $\left(0,0, \bar{u}_{1}, \bar{z}, \lambda\right) \in$ gph $\Phi$. Now, introduce the multiplier mapping

$$
\Lambda\left(u_{1}, u_{2}, z\right):=\left\{\lambda \in \mathbb{R}^{p} \mid \begin{array}{c}
\nabla_{z} f\left(u_{1}, z\right)-u_{2}+\mathcal{A}^{T} \lambda=0 \\
\mathcal{A} z+b \in \mathbb{R}_{-}^{p} \\
\lambda \in N_{\mathbb{R}_{-}^{p}}(\mathcal{A} z+b)
\end{array}\right\} .
$$

We show the claimed calmness of $\Psi$ in $(7.4)$ at $\left(0, \bar{u}_{1}, \bar{z}\right)$. Assume by contradiction the existence of sequences $\left(u_{2}^{(i)}, u_{1}^{(i)}, z^{(i)}\right) \rightarrow\left(0, \bar{u}_{1}, \bar{z}\right)$ with

$$
\left(u_{1}^{(i)}, z^{(i)}\right) \in \Psi\left(u_{2}^{(i)}\right) \quad \text { and } \quad d\left(\left(u_{1}^{(i)}, z^{(i)}\right), \Psi(0)\right) \geq i\left\|u_{2}^{(i)}\right\| \quad \forall i .
$$

By definition of $\Psi$, the first relation in (7.6) implies the existence of a sequence $\lambda^{(i)}$ with

$$
\left(u_{1}^{(i)}, z^{(i)}, \lambda^{(i)}\right) \in \Phi\left(u_{2}^{(i)}\right) \quad \forall i
$$

which in turn entails that $\left.\lambda^{(i)}\right) \in \Lambda\left(u_{1}^{(i)}, u_{2}^{(i)}, z^{(i)}\right)$ for all $i$ due to the equivalence

$$
\lambda \in \Lambda\left(u_{1}, u_{2}, z\right) \Leftrightarrow\left(u_{1}, z, \lambda\right) \in \Phi\left(u_{2}\right) .
$$

Next we observe that $\Lambda$ is upper Lipschitz at $\left(\bar{u}_{1}, 0, \bar{z}\right)$ (see, e.g., Lem. 4.44 and Rem. 4.45 in [2]). Since $\Lambda\left(\bar{u}_{1}, 0, \bar{z}\right)$ is compact (as a consequence of the our Slater point assumption 2.) it follows that for sufficiently large $i$ the multipliers belong to a bounded set. Consequently, we may pass to a subsequence which converges to some $\bar{\lambda}$. Now, the closeness of $\Phi$ along with (7.7) provides that $\left(\bar{u}_{1}, \bar{z}, \bar{\lambda}\right) \in \Phi(0)$, whence $\bar{\lambda} \in \Lambda\left(\bar{u}_{1}, 0, \bar{z}\right)$.

Next, we endow the Cartesian product $\mathbb{R}^{s+t} \times \mathbb{R}^{p}$ (containing the elements $\left(\left(u_{1}, z\right), \lambda\right)$ ) with a new norm $\left\|\left(\left(u_{1}, z\right), \lambda\right)\right\|_{*}:=\max \left\{\left\|\left(u_{1}, z\right)\right\|,\|\lambda\|\right\}$. Let $d_{*}$ be the distance function associated with $\|\cdot\|_{*}$ and put

$$
\mathcal{B}:=\bigcup_{\left(u_{1}, z\right) \in \Psi(0)} \Lambda\left(u_{1}, 0, z\right)
$$

Since $\Phi(0) \subseteq \Psi(0) \times \mathcal{B}$, one has by definition of $d_{*}$ that

$$
\begin{aligned}
d_{*}\left(\left(u_{1}^{(i)}, z^{(i)}, \lambda^{(i)}\right), \Phi(0)\right) & \geq d_{*}\left(\left(u_{1}^{(i)}, z^{(i)}, \lambda^{(i)}\right), \Psi(0) \times \mathcal{B}\right) \\
& =\inf _{(a, b, c) \in \Psi(0) \times \mathcal{B})} \max \left\{\left\|\left(u_{1}^{(i)}, z^{(i)}\right)-(a, b)\right\|,\left\|\lambda^{(i)}-c\right\|\right\} \\
& \geq \max \left\{\inf _{(a, b) \in \Psi(0)}\left\|\left(u_{1}^{(i)}, z^{(i)}\right)-(a, b)\right\|, \inf _{c \in \mathcal{B}}\left\|\lambda^{(i)}-c\right\|\right\} \\
& \geq d\left(\left(u_{1}^{(i)}, z^{(i)}\right), \Psi(0)\right) \geq i\left\|u_{2}^{(i)}\right\| \quad \forall i,
\end{aligned}
$$


where the last inequality is taken from the second part of (7.6). This, however, contradicts the already shown calmness of $\Phi$ at $\left(0,0, \bar{u}_{1}, \bar{z}, \lambda\right)$ where $\left(0,0, \bar{u}_{1}, \bar{z}, \lambda\right) \in \operatorname{gph} \Phi$.

Acknowledgements. We would like to thank an anonymous referee for his constructive remarks which helped to improve the presentation of this paper.

\section{REFERENCES}

[1] N. Biggs, Algebraic Graph Theory. Cambridge University Press, Cambrige, 2nd edition (1994).

[2] J.F. Bonnans and A. Shapiro, Perturbation Analysis of Optimization Problems. Springer, New York (2000).

[3] J.B. Cardell, C.C. Hitt and W.W. Hogan, Market power and strategic interaction in electricity networks. Resour. Energy Econ. 19 (1997) 109-137.

[4] S. Dempe, J. Dutta and S. Lohse, Optimality conditions for bilevel programming problems. Optimization 55 (2006) 505-524.

[5] A.L. Dontchev and R.T. Rockafellar, Characterization of strong regularity for variational inequalities over polyhedral convex sets. SIAM J. Optim. 7 (1996) 1087-1105.

[6] J.F. Escobar and A. Jofre, Monopolistic competition in electricity networks with resistance losses. Econ. Theor. 44 (2010) $101-121$.

[7] R. Henrion and W. Römisch, On M-stationary points for a stochastic equilibrium problem under equilibrium constraints in electricity spot market modeling. Appl. Math. 52 (2007) 473-494.

[8] R. Henrion, J. Outrata and T. Surowiec, On the coderivative of normal cone mappings to inequality systems. Nonlinear Anal. 71 (2009) 1213-1226.

[9] R. Henrion, B.S. Mordukhovich and N.M. Nam, Second-order analysis of polyhedral systems in finite and infinite dimensions with applications to robust stability of variational inequalities. SIAM J. Optim. 20 (2010) 2199-2227.

[10] B.F. Hobbs, Strategic gaming analysis for electric power systems: An MPEC approach. IEEE Trans. Power Syst. 15 (2000) 638-645.

[11] X. Hu and D. Ralph, Using EPECs to model bilevel games in restructured electricity markets with locational prices. Oper. Res. 55 (2007) 809-827.

[12] X. Hu, D. Ralph, E.K. Ralph, P. Bardsley and M.C. Ferris, Electricity generation with looped transmission networks: Bidding to an ISO. Research Paper No. 2004/16, Judge Institute of Management, Cambridge University (2004).

[13] D. Klatte and B. Kummer, Nonsmooth Equations in Optimization. Kluwer, Academic Publishers, Dordrecht (2002).

[14] D. Klatte and B. Kummer, Constrained minima and Lipschitzian penalties in metric spaces. SIAM J. Optim. 13 (2002) 619-633.

[15] Z.Q. Luo, J.S. Pang and D. Ralph, Mathematical programs with equilibrium constraints. Cambridge University Press, Cambridge (1996).

[16] B.S. Mordukhovich, Metric approximations and necessary optimality conditions for general classes of extremal problems. Soviet Mathematics Doklady 22 (1980) 526-530.

[17] B.S. Mordukhovich, Variational Analysis and Generalized Differentiation, Basic Theory 1, Applications 2. Springer, Berlin (2006).

[18] B.S. Mordukhovich and J. Outrata, On second-order subdifferentials and their applications. SIAM J. Optim. 12 (2001) 139-169.

[19] B.S. Mordukhovich and J. Outrata, Coderivative analysis of quasi-variational inequalities with applications to stability and optimization. SIAM J. Optim. 18 (2007) 389-412.

[20] J.V. Outrata, A generalized mathematical program with equilibrium constraints. SIAM J. Control Opt. 38 (2000) 1623-1638.

[21] J.V. Outrata, A note on a class of equilibrium problems with equilibrium constraints. Kybernetika 40 (2004) 585-594.

[22] J.V. Outrata, M. Kocvara and J. Zowe, Nonsmooth approach to optimization problems with equilibrium constraints. Kluwer Academic Publishers, Dordrecht (1998).

[23] S.M. Robinson, Some continuity properties of polyhedral multifunctions. Math. Program. Stud. 14 (1976) $206-214$.

[24] S.M. Robinson, Strongly regular generalized equations. Math. Oper. Res. 5 (1980) 43-62.

[25] R.T. Rockafellar and R.J.-B. Wets, Variational Analysis. Springer, Berlin (1998).

[26] V.V. Shanbhag, Decomposition and Sampling Methods for Stochastic Equilibrium Problems. Ph.D. thesis, Stanford University (2005).

[27] C.-L. Su, Equilibrium Problems with Equilibrium Constraints: Stationarities, Algorithms and Applications. Ph.D. thesis, Stanford University (2005).

[28] J.J. Ye and X.Y. Ye, Necessary optimality conditions for optimization problems with variational inequality constraints. Math. Oper. Res. 22 (1997) 977-997. 